\title{
Multi-Layer Gaussian-based Multi-Configuration Time-Dependent Hartree (ML-GMCTDH) Simulations of Ultrafast Charge Separation in a Donor-Acceptor \\ Complex
}

\author{
Francesco Di Maiolo, ${ }^{1, \text { a) }}$ Graham A. Worth, ${ }^{2, \text { b) }}$ and Irene Burghardt ${ }^{1, c)}$ \\ ${ }^{1)}$ Institute of Physical and Theoretical Chemistry, Goethe University Frankfurt, \\ Max-von-Laue-Str. 7, 60438 Frankfurt, Germany \\ ${ }^{2)}$ Department of Chemistry, University College London, 20, Gordon St., \\ London WC1H OAJ, UK
}

(Dated: 12 March 2021)

We report on first applications of the Multi-Layer Gaussian-based MultiConfiguration Time-Dependent Hartree (ML-GMCTDH) method [Römer et al., J. Chem. Phys. 138, 064106 (2013)], beyond its basic two-layer variant. The ML-GMCTDH scheme provides an embedding of a variationally evolving Gaussian wavepacket basis into a hierarchical tensor representation of the wavefunction. A first principles parametrized model Hamiltonian for ultrafast non-adiabatic dynamics in an oligothiophene-fullerene charge transfer complex is employed, relying on a two-state linear vibronic coupling model which combines a distribution of tuning type modes with an intermolecular coordinate that also modulates the electronic coupling. Efficient ML-GMCTDH simulations are carried out for up to 300 vibrational modes, using an implementation within the QUANTICS program. Excellent agreement with reference ML-MCTDH calculations is obtained.

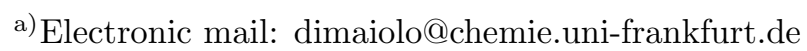

b)Electronic mail: g.a.worth@ucl.ac.uk

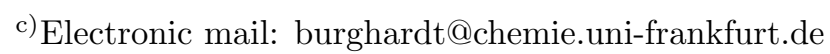




\section{INTRODUCTION}

Moving Gaussian basis sets are a highly attractive way of representing quantum evolution, combining a trajectory type representation with quantum non-locality. These methods have a long tradition in semiclassical dynamics and include classically evolving Gaussian wavepackets (GWPs), ${ }^{1,2}$ notably Heller's Thawed Gaussians (TGs) ${ }^{3}$ and Frozen Gaussians (FGs), ${ }^{4}$ variationally evolving GWPs, ${ }^{5}$ and variational GWP superpositions. ${ }^{6,7}$ Over recent years, many methods have been developed that are built on these basic concepts, e.g., the Full Multiple Spawning (FMS) approach, ${ }^{8}$ the Coupled Coherent States method, ${ }^{9}$ the multiconfigurational Ehrenfest approach, ${ }^{10,11}$ and the variational Multi-Configurational Gaussian (vMCG) method. ${ }^{12,13}$ An important area of application is on-the-fly dynamics, e.g., in the Ab Initio Multiple Spawning (AIMS) approach, ${ }^{14,15}$ the Direct Dynamics variational MultiConfigurational Gaussian (DD-vMCG) method, ${ }^{16-18}$ the $a b$ initio Multiple Cloning MultiConfigurational Ehrenfest (AIMC-MCE) approach, ${ }^{19}$ and the on-the-fly ab initio (OTF-AI) semiclassical dynamics method. ${ }^{20}$ Also, statistical treatments, ${ }^{21}$ system-bath problems, ${ }^{22}$ and quantum-classical hybrid approaches ${ }^{11,23}$ are naturally amenable to a GWP based treatment. Yet, in many applications, the accuracy of GWP based propagation is an issue, especially if the relevant dynamics are high-dimensional and correlated. Therefore, GWP based methods are often considered disjoint from numerically exact wavepacket propagation schemes.

In order to make Gaussian basis sets suitable for accurate quantum propagation in many dimensions, the combination of GWPs with hierarchical tensor representations of the wavefunction is a key step. The Gaussian-based Multi-Configuration Time-Dependent Hartree

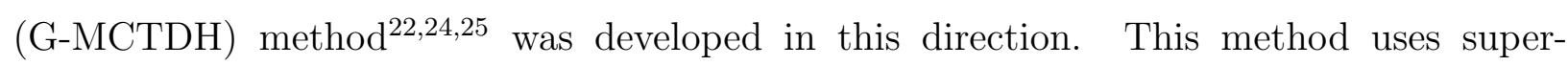
positions of Hartree products that can be either of "all-GWP" type or involve hybrid configurations where GWPs and MCTDH type ${ }^{26-28}$ particles are combined into a highdimensional wavefunctions. The vMCG scheme ${ }^{12,13,16}$ can be understood as a variant of GMCTDH, which uses the simplest form of GWP superpositions, in the absence of an MCTDH type structure of the wavefunction. Within the MCTDH family of methods, ${ }^{26-28}$ even more powerful schemes are the recently developed hierarchical multi-layer (ML-MCTDH) approaches ${ }^{29-32}$ which permit accurate wavefunction propagation for hundreds of degrees 
of freedom. Related methods include, e.g., time-dependent density matrix renormalization group (t-DMRG) approaches. ${ }^{33}$

In Ref. [34], a two-layer 2L-GMCTDH scheme was proposed which broadens the scope of GMCTDH by combining GWPs into flexible first-layer particles. Importantly, the variational freedom of the wavefunction construction allows the first layer to be orthogonal, even though the second layer is composed of non-orthogonal GWP functions. While multi-dimensional FGs are generally taken to be factorizable - and, hence, uncorrelated - these can be combined into correlated multidimensional first-layer modes. In Ref. [34], a natural continuation to an arbitrary number of layers was further proposed. Since orthogonality can be imposed for all layers except for the last, the overall scheme is entirely analogous to the ML-MCTDH method, ${ }^{29-32}$ except that the last layer is composed of GWPs, and the mean fields are adapted to the non-orthogonality of the latter.

While we previously implemented the hierarchical two-layer version of the method (2LGMCTDH) and presented applications to vibrational energy redistribution ${ }^{35}$ and nonadiabatic dynamics ${ }^{36}$ for up to 100 modes, the present paper presents the first application of a more general hierarchical ML-GMCTDH scheme with an arbitrary number of layers. Non-adiabatic dynamics calculations are carried out for a realistic donor-acceptor system comprising up to 300 modes. These calculations are benchmarked against highly accurate ML-MCTDH results. The ML-GMCTDH scheme has been implemented in the QUANTICS package. $^{37,38}$

We demonstrate the methodology for a two-state vibronic coupling model representing charge separation in a fullerene-oligothiophene donor-acceptor (DA) complex. This system is a minimal model for the description of exciton dissociation in a paradigm DA system of organic photovoltaics, composed of poly-3-hexylthiophene (P3HT) and phenyl- $\mathrm{C}_{61}$ butyric acid methyl ester (PCBM) components. ${ }^{39-41}$ The P3HT:PCBM bulk heterojunction material ${ }^{39}$ exhibits ultrafast charge separation dynamics, on a time scale of $\sim 50 \mathrm{fs}^{40,41}$ In a previous study ${ }^{42,43}$ we employed a Linear Vibronic Coupling (LVC) Hamiltonian in conjunction with the MCTDH method to describe the ultrafast, coherent charge transfer dynamics in this system. The Hamiltonian was parametrized by electronic structure calculations us- 
ing long-range corrected time-dependent density functional theory (LR-TDDFT) and the diabatization procedure described in Ref. [42], along with an ab initio generated spectral density of the phonon modes of the DA complex. In Refs. [44-46], extensions of this model to multiple electronic states are described.

The present model highlights the pronounced role of vibronic coupling effects in the charge separation at the DA interface. First, CC stretch type modes play a prominent role due to their large vibronic couplings (i.e., large Huang-Rhys factors ${ }^{47}$ ); these modes typically induce curve crossing dynamics. Second, a dense frequency distribution is essential, and vibronic resonance effects ${ }^{48-50}$ could be involved. Third, an intermolecular mode is included which modulates the electronic coupling, and leads to a smoothing of the population decay. ${ }^{43}$ The resulting dynamics bears vibronically coherent features, as observed experimentally, ${ }^{40,51}$ but at the same time shows that an effectively irreversible transfer is taking place.

Since the observed dynamics are strongly correlated, a multiconfigurational treatment is necessary. More approximate methods like Ehrenfest dynamics fail entirely for this system, ${ }^{11}$ while Multi-Configuration Ehrenfest (MCE) approaches lead to slow convergence for a limited number of modes (typically for 10-40 modes). ${ }^{11,52}$ For similar numbers of modes, vMCG dynamics are difficult to converge, too, while the G-MCTDH approach works well, but becomes rapidly expensive. ${ }^{52}$ Using $2 \mathrm{~L}-\mathrm{GMCTDH}$, converged calculations could be performed up to 100 modes.$^{36}$ Here, we go significantly beyond these previous studies and demonstrate the application of the ML-GMCTDH approach for up to 300 modes. To obtain a series of realizations of the linear vibronic coupling Hamiltonian of Ref. [43], the relevant spectral density was rediscretized, using a variable number of harmonic modes.

The remainder of the manuscript is organized as follows: Sec. II summarizes the wavefunction ansatz and equations of motion of the ML-GMCTDH method. Sec. III presents the charge transfer system under study. Sec. IV summarizes the simulation set-up, Sec. V presents the results obtained for this system, and Sec. VI concludes. 


\section{MULTI-LAYER GAUSSIAN-BASED MCTDH (ML-GMCTDH) METHOD}

The multi-layer ML-GMCTDH method can be understood from two perspectives. In Ref. [34], the approach was introduced as a natural extension of the two-layer 2L-GMCTDH method $^{34-36}$ to a larger number of layers. Alternatively, one can think of a modified MLMCTDH approach, ${ }^{29-32}$ where GWPs replace the single-particle functions (SPFs) of the last layer. Here, we will follow the former approach which highlights the aspect that the nonorthogonal GWP representation is embedded into a flexible, orthogonal SPF representation. From both perspectives, the result is a multi-layer method which is identical to ML-MCTDH except for the last layer, which is composed of GWPs. In the following, we therefore first address the 2L-GMCTDH approach, and then present its generalization to the ML-GMCTDH scheme.

\section{A. Two-Layer G-MCTDH}

Here, we address the basic two-layer scheme ${ }^{34}$ which best illustrates the general multi-layer concept, motivated by the limitations of the FG-based wavefunction ansatz that is commonly employed in the G-MCTDH ${ }^{22,24,25}$ method and its vMCG variant. ${ }^{12,13,16}$ Multidimensional FGs are uncorrelated product functions of fixed width and therefore far less suitable to describe correlated dynamics than typical SPFs within the MCTDH approach. ${ }^{26-28}$ In order to re-introduce flexibility and correlations into FG based wavefunctions, it was suggested in Ref. [34] to create a new type of SPFs defined in terms of superpositions of FGs. This strategy entails a two-layer representation.

Since we will address coupled electronic states, we describe combined electronic-vibrational representations of the wavefunction. Specifically, we will employ the so-called single-set scheme, ${ }^{28,36}$ where the GWP basis is taken to be state-independent, and the electronic states are treated as a set of additional electronic SPFs which are here assumed to be timeindependent. While several types of representations - single-set, multi-set, and a hybrid variant - were implemented in the $2 \mathrm{~L}-\mathrm{GMCTDH}$ framework, ${ }^{36}$ we focus on the single-set 
variant in the present context since this is the approach chosen in the ML-MCTDH method (see also the discussion of this aspect below).

In the formal development of this section, we will refer to a diabatic Hamiltonian of the general form ${ }^{53}$

$$
\widehat{H}=\sum_{s}^{n_{s}} \sum_{s^{\prime}}^{n_{s}} \widehat{H}^{\left(s s^{\prime}\right)}|s\rangle\left\langle s^{\prime}\left|=\widehat{T} \widehat{\mathbf{1}}+\sum_{s}^{n_{s}} \sum_{s^{\prime}}^{n_{s}} \widehat{V}^{\left(s s^{\prime}\right)}\right| s\right\rangle\left\langle s^{\prime}\right|
$$

where the kinetic energy term is electronically diagonal while electronic couplings appear in the form of potential type matrix elements (rather than the derivative couplings pertaining to the adiabatic picture). ${ }^{53}$ In Sec. III, the specific vibronic coupling Hamiltonian employed in this paper will be described in detail.

\section{Wavefunction ansatz}

The general wavefunction ansatz remains the same as in the MCTDH method, ${ }^{28,34}$

$$
\begin{aligned}
|\Psi(\mathbf{x}, t)\rangle & =\sum_{s}^{n_{s}} \sum_{J} A_{J s}^{(1)}(t) \Phi_{J}(\mathbf{x}, t)|s\rangle \\
& =\sum_{s}^{n_{s}} \sum_{J} A_{J s}^{(1)}(t) \prod_{\kappa=1}^{f} \chi_{j_{\kappa}}^{(\kappa)}\left(\mathbf{x}_{\kappa}, t\right)|s\rangle
\end{aligned}
$$

with time-dependent coefficients $A_{J s}^{(1)}(t)$, time-dependent SPFs $\chi_{j_{\kappa}}^{(\kappa)}\left(\mathbf{x}_{\kappa}, t\right)$, where $\mathbf{x}_{\kappa}=$ $\left(x_{1}, x_{2}, \ldots, x_{d_{\kappa}}\right)$ generally represents a multidimensional ("combined") mode, and the electronic states $|s\rangle, s=1, \ldots, n_{s}$. The multi-index $J=\left(j_{1}, j_{2}, \ldots, j_{f}\right)$ collects all SPF indices for a given configuration $\Phi_{J}$; here, $j_{\kappa}=1,2, \ldots, n^{(\kappa)}$, where $n^{(\kappa)}$ defines the dimension of the SPF basis for the $\kappa$ th mode. The coefficients $A_{J s}^{(1)}$ are labelled with the superscript index (1) to indicate that these quantities belong to the first layer of the wavefunction representation. In the single-set 2L-GMCTDH approach, ${ }^{36}$ which we focus on here, the SPFs $\chi_{j_{\kappa}}^{(\kappa)}$ are state-independent and the electronic degrees of freedom are treated as an independent set of additional electronic SPFs. The latter are defined as time-independent and identical to the electronic states $|s\rangle$, such that the number of electronic SPFs corresponds to the number $n_{s}$ of electronic states. 
Differently from MCTDH, the first-layer SPFs $\chi_{j_{\kappa}}^{(\kappa)}$ of Eq. (2) are now defined as superpositions of GWPs, ${ }^{34-36}$

$$
\begin{aligned}
\chi_{j}^{(\kappa)}\left(\mathbf{x}_{\kappa}, t\right) & =\sum_{L} A_{j, L}^{(2, \kappa)}(t) G_{L}^{(\kappa)}\left(\mathbf{x}_{\kappa}, t\right) \\
& =\sum_{L} A_{j, L}^{(2, \kappa)}(t) \prod_{\mu=1}^{f^{(\kappa)}} g_{l_{\mu}}^{(\kappa, \mu)}\left(\mathbf{x}_{\kappa, \mu}, t\right)
\end{aligned}
$$

with multi-indices $L=\left(l_{1}, l_{2}, \ldots, l_{f^{\kappa}}\right)$, where $l_{\mu}=1,2, \ldots, n^{(\kappa, \mu)}$, second-layer coefficients $A_{j, L}^{(2, \kappa)}(t)$, and multi-dimensional GWPs $g_{l}^{(\kappa, \mu)}$.

$$
\begin{aligned}
& g_{l}^{(\kappa, \mu)}\left(\mathbf{x}_{\kappa, \mu}, t\right)=g_{l}^{(\kappa, \mu)}\left(\mathbf{x}_{\kappa, \mu}, \boldsymbol{\Lambda}_{l}^{(\kappa, \mu)}(t)\right) \\
& \quad=\exp \left[\mathbf{x}_{\kappa, \mu}^{T} \boldsymbol{a}_{l}^{(\kappa, \mu)} \mathbf{x}_{\kappa, \mu}+\left(\boldsymbol{\xi}_{l}^{(\kappa, \mu)}(t)\right)^{T} \mathbf{x}_{\kappa, \mu}+\eta_{l}^{(\kappa, \mu)}(t)\right]
\end{aligned}
$$

whose time evolution is defined by the parameter set $\boldsymbol{\Lambda}_{l}^{(\kappa, \mu)}(t)=\left(\boldsymbol{a}_{l}^{(\kappa, \mu)}, \boldsymbol{\xi}_{l}^{(\kappa, \mu)}(t), \eta_{l}^{(\kappa, \mu)}(t)\right)$. Since we focus on FGs, the symmetric width matrix $\boldsymbol{a}_{l}^{(\kappa, \mu)}$ is taken to be constant, realvalued and time-independent. The complex parameters $\boldsymbol{\xi}_{l}^{(\kappa, \mu)}=-2 \boldsymbol{a}_{l}^{(\kappa, \mu)} \boldsymbol{q}_{l}^{(\kappa, \mu)}+i \boldsymbol{p}_{l}^{(\kappa, \mu)}$ label a time-evolving phase-space point with center position $\boldsymbol{q}_{l}^{(\kappa, \mu)}$ and center momentum $\boldsymbol{p}_{l}^{(\kappa, \mu)}$ in the $(\kappa, \mu)$ th subspace. Finally, the real part of $\eta_{l}^{(\kappa, \mu)}$ fixes the norm of the FG and the imaginary part of $\eta_{l}^{(\kappa, \mu)}$ is a phase factor. The latter is set to zero in the following, $\operatorname{Im}\left(\eta_{l}^{(\kappa, \mu)}\right)$ $=0$, such that the phase information is effectively absorbed into the $A_{j, L}^{(2, \kappa)}(t)$ coefficients; this corresponds to a "coherent state gauge". ${ }^{54,55}$ As a result, the following factorized form of the multi-dimensional FGs is obtained,

$$
\begin{aligned}
& g_{l}^{(\kappa, \mu)}\left(\mathbf{x}_{\kappa, \mu}, t\right)=\prod_{j} N_{l j}^{(\kappa, \mu)} \\
& \quad \times \exp \left[\left(\boldsymbol{a}_{l}^{(\kappa, \mu)}\right)_{j j}\left(\mathbf{x}_{\kappa, \mu}\right)_{j}^{2}+\left(\boldsymbol{\xi}_{l}^{(\kappa, \mu)}\right)_{j}(t)\left(\mathbf{x}_{\kappa, \mu}\right)_{j}\right]
\end{aligned}
$$

with the overall normalization constant $N_{l}^{(\kappa, \mu)}=\prod_{j} N_{l j}^{(\kappa, \mu)}$ where $N_{l j}^{(\kappa, \mu)}$ corresponds to the normalization for each 1D component GWP wavefunction,

$$
N_{l j}^{(\kappa, \mu)}=\left(-\frac{2 \operatorname{Re}\left(\boldsymbol{a}_{l}^{(\kappa, \mu)}\right)_{j j}}{\pi}\right)^{1 / 4} \exp \left(\frac{\left(\operatorname{Re}\left(\boldsymbol{\xi}_{l}^{(\kappa, \mu)}\right)_{j}\right)^{2}}{4 \operatorname{Re}\left(\boldsymbol{a}_{l}^{(\kappa, \mu)}\right)_{j j}}\right)
$$


To summarize, Eq. (2) and Eq. (3) define a generalized, two-layered G-MCTDH ansatz which has far more flexibility than the G-MCTDH reference method. The first-layer SPFs $\chi_{j}^{(\kappa)}$ represent time-evolving super-particles, defined by the time-evolving $A_{j, L}^{(2, \kappa)}$ coefficients and a set of GWP modes $g_{l}^{(\kappa, \mu)}$. These now appear in the second layer and are uniquely specified by the index pair $(\kappa, \mu)$, where $\mu=1,2, \ldots, f^{(\kappa)}$, labels the GWP subspaces within the first-layer $\kappa$ th subspace. Within the second-layer subspaces, $l=1, \ldots, n^{(\kappa, \mu)}$ runs over the basis set of second-layer GWPs $g_{l}^{(\kappa, \mu)}$, such that $n^{(\kappa, \mu)}$ GWPs per subspace contribute to the product configurations $G_{L}^{(\kappa)}$ of Eq. (3).

Importantly, this wavefunction representation permits the same gauge for the first layer as in standard MCTDH, i.e., within each $\kappa$ th first-layer subspace, the SPFs can be chosen as orthonormal at all times,

$$
\left\langle\chi_{j}^{(\kappa)}(t) \mid \chi_{j^{\prime}}^{(\kappa)}(t)\right\rangle=\delta_{j j^{\prime}}
$$

Within each $\kappa$ th subspace, a unitary evolution under a constraint operator $\widehat{h}_{c}^{(\kappa)}$ is allowed, ${ }^{28,34}$

$$
\widehat{P}^{(\kappa)}(t)\left(i \partial_{t}-\widehat{h}_{c}^{(\kappa)}\right)\left|\chi_{j}^{(\kappa)}(t)\right\rangle=|0\rangle
$$

with the time-dependent projection operator onto the $\kappa$ th subspace,

$$
\widehat{P}^{(\kappa)}(t)=\sum_{j}\left|\chi_{j}^{(\kappa)}(t)\right\rangle\left\langle\chi_{j}^{(\kappa)}(t)\right|
$$

In the following, the constraint operator $\widehat{h}_{c}^{(\kappa)}$ of Eq. (8) is set to zero, without loss of generality.

The conditions of Eq. (7) are preserved by the dynamics of the coefficients $A_{J s}^{(1)}$ and $A_{j, L}^{(2, \kappa)}$, despite the non-orthogonality of the GWP basis appearing in the second layer. Thus the firstlayer SPFs $\chi_{j}^{(\kappa)}$ remain orthonormal at all times, while the second-layer GWPs $g_{l}^{(\kappa, \mu)}$ remain normalized but non-orthogonal. An exception is the trivial case where $\chi_{j}^{(\kappa)}(t)=g_{j}^{(\kappa)}(t)$ such that the relevant first-layer SPF reduces to a single GWP and the second layer is absent. (As a result, the first-layer configurations are then of hybrid SPF/GWP type as in the G-MCTDH method. ${ }^{22,24,25}$ ) 
Finally, the wavefunction Eq. (2) can be expressed as follows in terms of sums over products of first-layer or second-layer SPFs and single-hole functions (SHFs),

$$
|\Psi\rangle=\sum_{j} \chi_{j}^{(\kappa)}\left|\psi_{j}^{(\kappa)}\right\rangle=\sum_{l} g_{l}^{(\kappa, \mu)}\left|\psi_{l}^{(\kappa, \mu)}\right\rangle
$$

where $\left|\psi_{j}^{(\kappa)}\right\rangle$ and $\left|\psi_{l}^{(\kappa, \mu)}\right\rangle$ refer to first-layer and second-layer SHFs, respectively,

$$
\begin{aligned}
\left|\psi_{j}^{(\kappa)}\right\rangle & =\sum_{s} \sum_{J^{(\kappa)}} A_{J^{(\kappa: j)} s}^{(1)} \Phi_{J^{(\kappa)}}|s\rangle, \\
\left|\psi_{l}^{(\kappa, \mu)}\right\rangle & =\sum_{j}\left[\sum_{L^{(\mu)}} A_{j, L^{(\mu) l)}}^{(2, \kappa)} G_{L^{(\mu)}}^{(\kappa)}\right]\left|\psi_{j}^{(\kappa)}\right\rangle
\end{aligned}
$$

whose overlap defines the first-layer and second-layer single-particle density matrices, $\rho_{j j^{\prime}}^{(\kappa)}=$ $\left\langle\psi_{j^{\prime}}^{(\kappa)} \mid \psi_{j}^{(\kappa)}\right\rangle$ and $\rho_{l l^{\prime}}^{(\kappa, \mu)}=\left\langle\psi_{l^{\prime}}^{(\kappa, \mu)} \mid \psi_{l}^{(\kappa, \mu)}\right\rangle$. In Eq. (11), we again used a shorthand notation for multi-indices, i.e., $J^{(\kappa)}=\left(j_{1}, \ldots, j_{\kappa-1}, j_{\kappa+1}, \ldots, j_{f}\right)$ and $J^{(\kappa: j)}=\left(j_{1}, \ldots, j_{\kappa-1}, j, j_{\kappa+1}, \ldots, j_{f}\right)$. Further, single-hole configurations are defined as $\Phi_{J^{(\kappa)}}=\prod_{\kappa^{\prime} \neq \kappa} \chi_{j_{\kappa^{\prime}}}^{\left(\kappa^{\prime}\right)}$. The second-layer quantities $L^{(\mu)}, L^{(\mu: l)}$ and $G_{L^{(\mu)}}^{(\kappa)}$ are defined analogously.

\section{Equations of motion}

In the variational equations of motion (EOMs), obtained from the Dirac-Frenkel variational principle, ${ }^{28,56}$ the electronic state index appears explicitly in the EOMs for the first-layer coefficients $\boldsymbol{A}^{(1)}$,

$$
i \dot{\boldsymbol{A}}_{s}^{(1)}=\sum_{s^{\prime}} \boldsymbol{H}^{\left(s s^{\prime}\right)} \boldsymbol{A}_{s^{\prime}}^{(1)}
$$

where the $\boldsymbol{A}^{(1)}$ vector has elements $A_{J s}^{(1)} \equiv\left(\boldsymbol{A}_{s}^{(1)}\right)_{J}$ and the Hamiltonian matrix elements in the basis of first-layer configurations read

$$
H_{J J^{\prime}}^{\left(s s^{\prime}\right)}=\left\langle\Phi_{J}\left|\widehat{H}^{\left(s s^{\prime}\right)}\right| \Phi_{J^{\prime}}\right\rangle
$$

Conversely, the equations for the second-layer coefficients $\boldsymbol{A}^{(2)}$ do not carry an electronic state index within the single-set scheme,

$$
i \boldsymbol{S}^{(\kappa)} \dot{\boldsymbol{A}}^{(2, \kappa)}=\left[\boldsymbol{H}^{(\kappa)}-i \boldsymbol{\tau}^{(\kappa)}\right] \boldsymbol{A}^{(2, \kappa)}
$$


In Eq. (14), all quantities are defined by analogy with the G-MCTDH method ${ }^{24}$ including the overlap matrix $\boldsymbol{S}^{(\kappa)}$, the differential overlap matrix $\boldsymbol{\tau}^{(\kappa)}$, and the effective mean-field Hamiltonian $\boldsymbol{H}^{(\kappa)}$ matrix,

$$
\begin{aligned}
& S_{j L, j^{\prime} L^{\prime}}^{(\kappa)}=\delta_{j j^{\prime}}\left\langle G_{L}^{(\kappa)} \mid G_{L^{\prime}}^{(\kappa)}\right\rangle, \quad \tau_{j L, j^{\prime} L^{\prime}}^{(\kappa)}=\delta_{j j^{\prime}}\left\langle G_{L}^{(\kappa)} \mid \partial_{t} G_{L^{\prime}}^{(\kappa)}\right\rangle \\
& H_{j L, j^{\prime} L^{\prime}}^{(\kappa)}=\left\langle G_{L}^{(\kappa)}\left|\left(1-\widehat{P}^{(\kappa)}\right)\left[\left(\boldsymbol{\rho}^{(\kappa)}\right)^{-1} \widehat{\boldsymbol{H}}^{(\kappa)}\right]_{j j^{\prime}}\right| G_{L^{\prime}}^{(\kappa)}\right\rangle
\end{aligned}
$$

Summations over the electronic states appear indirectly in the definitions of the singleparticle density $\rho_{j j^{\prime}}^{(\kappa)}=\left\langle\psi_{j^{\prime}}^{(\kappa)} \mid \psi_{j}^{(\kappa)}\right\rangle$, using Eq. (11), and the mean-field Hamiltonian of Eq. $(16)$

$$
\widehat{H}_{l l^{\prime}}^{(\kappa)}=\sum_{s} \sum_{s^{\prime}}\left\langle\psi_{l}^{(\kappa)}\left|\widehat{H}^{\left(s s^{\prime}\right)}\right| \psi_{l^{\prime}}^{(\kappa)}\right\rangle
$$

Finally, the EOMs for the GWP parameters $\Lambda^{(\kappa, \mu)}$ also have the same structure as in G$\mathrm{MCTDH},{ }^{24}$ except that these equations now appear in the second layer,

$$
i \boldsymbol{C}^{(\kappa, \mu)} \dot{\Lambda}^{(\kappa, \mu)}=\boldsymbol{Y}^{(\kappa, \mu)}
$$

In Eq. (18), the $\boldsymbol{C}$ matrix and $\boldsymbol{Y}$ vector are defined as

$$
\begin{gathered}
C_{l \alpha, l^{\prime} \beta}^{(\kappa, \mu)}=\rho_{l l^{\prime}}^{(\kappa, \mu)}\left\langle\partial_{\alpha} g_{l}^{(\kappa, \mu)}\left|\left(1-\widehat{P}^{(\kappa, \mu)}\right)\right| \partial_{\beta} g_{l^{\prime}}^{(\kappa, \mu)}\right\rangle \\
Y_{l \alpha}^{(\kappa, \mu)}=\sum_{l^{\prime}}\left\langle\partial_{\alpha} g_{l}^{(\kappa, \mu)}\left|\left(1-\widehat{P}^{(\kappa, \mu)}\right) \widehat{H}_{l l^{\prime}}^{(\kappa, \mu)}\right| g_{l^{\prime}}^{(\kappa, \mu)}\right\rangle
\end{gathered}
$$

where we used the shorthand notation $\partial_{\alpha} g_{l}^{(\kappa, \mu)}=\left(\partial g_{l}^{(\kappa, \mu)} / \partial \Lambda_{l, \alpha}^{(\kappa, \mu)}\right)$, and where the secondlayer mean-field Hamiltonian matrix elements are given as

$$
\widehat{H}_{l l^{\prime}}^{(\kappa, \mu)}=\sum_{s} \sum_{s^{\prime}}\left\langle\psi_{l}^{(\kappa, \mu)}\left|\widehat{H}^{\left(s s^{\prime}\right)}\right| \psi_{l^{\prime}}^{(\kappa, \mu)}\right\rangle
$$

To summarize, the EOMs of the 2L-GMCTDH method combine (i) a single-set MCTDHlike equation for the first-layer coefficients $\boldsymbol{A}^{(1)}$ (Eq. (12)), (ii) a G-MCTDH-like equation 
for the second-layer coefficients $\boldsymbol{A}^{(2)}$ (Eq. (14)), and (iii) a G-MCTDH like equation for the GWP parameters $\boldsymbol{\Lambda}^{(\kappa, \mu)}$ (Eq. (18)). This scheme is superior to G-MCTDH because the GWP parameter evolution is now restricted to low-dimensional $(\kappa, \mu)$ subspaces; this alleviates the unfavorable, cubic scaling $\sim\left(n^{(\kappa, \mu)} d_{\kappa, \mu}\right)^{3}$ of FG propagation as a function of the number of combined degrees of freedom $d_{\kappa, \mu}$ and the number $n^{(\kappa, \mu)}$ of GWPs in the $(\kappa, \mu)$ th subspace. These equations are discussed in more detail in Refs. [34-36].

\section{B. Multi-Layer GMCTDH}

The generalization of the above ansatz to more than two layers is conceptually straightforward: The upper, orthogonal layer of the 2L-GMCTDH wavefunction can be expanded into multiple layers - as in the ML-MCTDH method $^{29-32}$ - while the last layer remains of GWP type. This formal development has been carried out in Ref. [34] and is recapitulated in the following.

As mentioned above, the single-set approach ${ }^{28,36}$ is employed, which is the standard approach within the ML-MCTDH scheme. ${ }^{32}$ While the single-set scheme may offer less flexibility than multi-set or hybrid schemes, ${ }^{36}$ it is more straightforward to implement in a general multilayer context, and convergence is ensured by the flexibility of the hierarchical wavefunction form.

\section{Wavefunction ansatz}

As in Sec. II A, we start with the wave function $|\Psi\rangle$ in single-set form,

$$
\begin{aligned}
|\Psi(t)\rangle & =\sum_{s}^{n_{s}} \sum_{J} A_{J s}^{(1)}(t) \Phi_{J}^{(1)}(t)|s\rangle \\
& =\sum_{s}^{n_{s}} \sum_{J} A_{J s}^{(1)}(t) \prod_{\kappa_{1}=1}^{f^{(1)}} \chi_{\kappa_{\kappa_{1}}}^{\left(1, \kappa_{1}\right)}(t)|s\rangle
\end{aligned}
$$

where the notation is entirely analogous to Eq. (2), except that the configurations and SPFs are labeled with a superscript index (1) to underscore that these belong to the first 
layer of the wavefunction. Now the first-layer SPFs $\left\{\chi_{j_{\kappa_{1}}}^{\left(1, \kappa_{1}\right)}\right\}$ are expanded into secondlayer orthogonal configurations, and likewise for successive layers. However, the last layer is represented by non-orthogonal GWPs as in the 2L-GMCTDH scheme.

For an $M$-layer ansatz, the SPFs of the first $(M-1)$ layers are expanded as follows,

$$
\begin{aligned}
\chi_{j}^{\left(m, \nu_{m}\right)}(t) & =\sum_{J} A_{j, J}^{\left(m+1, \nu_{m}\right)}(t) \Phi_{J}^{\left(m+1, \nu_{m}\right)}(t) \\
& =\sum_{J} A_{j, J}^{\left(m+1, \nu_{m}\right)}(t) \prod_{\kappa_{m+1}=1}^{f_{\nu_{m}}^{(m+1)}} \chi_{j_{\kappa_{m}+1}}^{\left(m+1, \nu_{m+1}\right)}(t)
\end{aligned}
$$

where $m=1,2, \ldots, M-1$ and multi-indices are employed as above. Further, we introduced the shorthand notation $\nu_{m}=\left(\kappa_{1}, \kappa_{2}, \ldots, \kappa_{m}\right)$ in order to label a particular $m$ th-layer mode. (If $m=1$, we obtain $\nu_{1}=\kappa_{1}$, consistent with Eq. (22).) That is, for a given $m$ th-layer mode, $\nu_{m}$ uniquely defines the full position in the wavefunction expansion, while the active index in the $m$ th layer is $\kappa_{m}=1,2, \ldots, f_{\nu_{m-1}}^{(m)}$. Finally, the SPFs of the final ( $M$ th) layer are FG functions, that is

$$
\Phi_{J}^{\left(M, \nu_{M-1}\right)}(t) \equiv G_{J}^{\left(M, \nu_{M-1}\right)}(t)=\prod_{\kappa_{M}=1}^{f_{\nu_{M}}^{(M)}} g_{j}^{\left(M, \nu_{M}\right)}\left(\boldsymbol{\Lambda}_{j}^{\left(M, \nu_{M}\right)}(t)\right)
$$

that is, $\chi_{j}^{\left(M, \nu_{M}\right)}(t)=g_{j}^{\left(M, \nu_{M}\right)}\left(\boldsymbol{\Lambda}_{j}^{\left(M, \nu_{M}\right)}(t)\right)$.

As in the 2L-GMCTDH case, the representations of Eq. (22) and Eq. (23) are not unique, and we will use the standard gauge conventions throughout:

(i) For the first $M-1$ layers, the initial SPFs $\chi^{\left(m, \nu_{m}\right)}(0)$ are chosen mutually orthonormal and their time evolution inside the $\nu_{m}$ th subspaces is fixed by the condition (see Eq. (8))

$$
\widehat{P}^{\left(m, \nu_{m}\right)}\left(i \partial_{t}-\widehat{h}_{c}^{\left(m, \nu_{m}\right)}\right)\left|\chi_{j}^{\left(m, \nu_{m}\right)}\right\rangle=|0\rangle
$$

where $\widehat{P}^{\left(m, \nu_{m}\right)}$ is the orthogonal projector on the $\nu_{m}$ th subspace,

$$
\widehat{P}^{\left(m, \nu_{m}\right)}=\sum_{j}\left|\chi_{j}^{\left(m, \nu_{m}\right)}\right\rangle\left\langle\chi_{j}^{\left(m, \nu_{m}\right)}\right|
$$


The constraint operator $\widehat{h}_{c}^{\left(m, \nu_{m}\right)}$ is an arbitrary Hermitian operator which guarantees that the SPFs stay orthonormal for all times:

$$
\left\langle\chi_{j}^{\left(m, \nu_{m}\right)}(t) \mid \chi_{j^{\prime}}^{\left(m, \nu_{m}\right)}(t)\right\rangle=\delta_{j j^{\prime}}
$$

In the following, we will again set $\widehat{h}_{c}^{\left(m, \nu_{m}\right)}=0$ as the simplest possible choice.

(ii) The GWPs $g_{j}^{\left(M, \nu_{M}\right)}\left(\boldsymbol{\Lambda}_{j}^{\left(M, \nu_{M}\right)}(t)\right)$ that replace the conventional SPFs in the $M$ th layer are chosen normalized, and their free phase factors are set to zero, $\operatorname{Im}\left(\eta_{j}^{\left(M, \nu_{M}\right)}\right)(t)=0$. The GWPs remain normalized but non-orthogonal throughout the propagation.

As above, it is convenient to define the $m$ th-layer single hole functions $\psi_{j}^{\left(m, \nu_{m}\right)}$,

$$
|\Psi\rangle=\sum_{j} \chi_{j}^{\left(m, \nu_{m}\right)}\left|\psi_{j}^{\left(m, \nu_{m}\right)}\right\rangle
$$

and using these, the SHF overlaps that define the $m$ th-layer density matrices,

$$
\rho_{j j^{\prime}}^{\left(m, \nu_{m}\right)}=\left\langle\psi_{j^{\prime}}^{\left(m, \nu_{m}\right)} \mid \psi_{j}^{\left(m, \nu_{m}\right)}\right\rangle,
$$

and the $m$ th-layer mean field Hamiltonian operators,

$$
\widehat{H}_{j j^{\prime}}^{\left(m, \nu_{m}\right)}=\left\langle\psi_{j}^{\left(m, \nu_{m}\right)}|\widehat{H}| \psi_{j^{\prime}}^{\left(m, \nu_{m}\right)}\right\rangle
$$

In the $M$ th layer, the overlap of GWP configurations is given as follows,

$$
S_{J J^{\prime}}^{\left(M, \nu_{M-1}\right)}=\left\langle G_{J}^{\left(M, \nu_{M-1}\right)} \mid G_{J^{\prime}}^{\left(M, \nu_{M-1}\right)}\right\rangle
$$

and the corresponding differential overlaps read

$$
\tau_{J J^{\prime}}^{\left(M, \nu_{M-1}\right)}=\left\langle G_{J}^{\left(M, \nu_{M-1}\right)} \mid \partial_{t} G_{J^{\prime}}^{\left(M, \nu_{M-1}\right)}\right\rangle .
$$

As a note on the above presentation, we assumed that all first-layer particles are subsequently expanded into $M$ layers. However, as will be illustrated below, a general ML-GMCTDH wavefunction can contain branches with variable numbers $M$ of layers, analogously to the general ML-MCTDH construction scheme. ${ }^{29-32}$ This includes the trivial case $M=1$ for selected branches where GWP particles are found in the first layer, $\chi_{j}^{(1, \kappa)}=g_{j}^{(1, \kappa)}$. 


\section{Equations of motion}

Using the Dirac-Frenkel variational principle, ${ }^{28,56}$ EOMs are now obtained for the coefficients of all layers, along with the GWP parameters of the last layer. To start with, the equation for the first-layer coefficients reads as Eq. (12),

$$
i \dot{\boldsymbol{A}}_{s}^{(1)}=\sum_{s^{\prime}} \boldsymbol{H}^{\left(1, s s^{\prime}\right)} \boldsymbol{A}_{s^{\prime}}^{(1)}
$$

where $\left(\boldsymbol{A}_{s}^{(1)}\right)_{J}=A_{J s}^{(1)}$, and the Hamiltonian in the basis of first-layer configurations is given as

$$
\boldsymbol{H}_{J, J^{\prime}}^{\left(1, s s^{\prime}\right)}=\left\langle\Phi_{J}^{(1)}\left|\widehat{H}^{\left(1, s s^{\prime}\right)}\right| \Phi_{J^{\prime}}^{(1)}\right\rangle
$$

Within the single-set scheme as defined in the ansatz Eq. (22), the electronic state indices exclusively appear in the first layer.

For the coefficients of the next $M-2$ layers we obtain the same equations of motion as in standard multi-layer MCTDH, ${ }^{30-32,57}$ i.e. for $m=2, \ldots, M-1$

$$
i \dot{\boldsymbol{A}}^{\left(m, \nu_{m}-1\right)}=\boldsymbol{H}^{\left(m, \nu_{m}-1\right)} \boldsymbol{A}^{\left(m, \nu_{m-1}\right)}
$$

with

$$
\begin{aligned}
H_{j J, j^{\prime} J^{\prime}}^{\left(m, \nu_{m}-1\right)}= & \left\langle\Phi_{J}^{\left(m, \nu_{m-1}\right)}\right|\left(1-\widehat{P}^{\left(m-1, \nu_{m-1}\right)}\right) \\
& \times\left[\left(\boldsymbol{\rho}^{\left(m-1, \nu_{m-1}\right)}\right)^{-1} \widehat{\boldsymbol{H}}^{\left(m-1, \nu_{m-1}\right)}\right]_{j j^{\prime}}\left|\Phi_{J^{\prime}}^{\left(m, \nu_{m-1}\right)}\right\rangle
\end{aligned}
$$

where $\widehat{\boldsymbol{H}}^{\left(m-1, \nu_{m-1}\right)}$ is the mean-field Hamiltonian operator pertaining to the $(m-1)$ st layer, see Eq. (30).

In the final ( $M$ th) layer, the equations of motion for the coefficients are the same as in the second layer of the 2L-GMCTDH variant, Eq. (14),

$$
i \boldsymbol{S}^{\left(M, \nu_{M-1}\right)} \dot{\boldsymbol{A}}^{\left(M, \nu_{M}-1\right)}=\left[\boldsymbol{H}^{\left(M, \nu_{M-1}\right)}-\boldsymbol{\tau}^{\left(M, \nu_{M-1}\right)}\right] \boldsymbol{A}^{\left(M, \nu_{M-1}\right)}
$$


with the $M$ th-layer effective mean-field Hamiltonian matrix

$$
\begin{aligned}
& H_{j J, j^{\prime} J^{\prime}}^{\left(M, \nu_{M-1}\right)}=\left\langle G_{J}^{\left(M, \nu_{M-1}\right)}\right|\left(1-\widehat{P}^{\left(M-1, \nu_{M-1}\right)}\right) \\
& \times\left[\left(\boldsymbol{\rho}^{\left(M-1, \nu_{M-1}\right)}\right)^{-1} \widehat{\boldsymbol{H}}^{\left(M-1, \nu_{M-1}\right)}\right]_{j j^{\prime}}\left|G_{J^{\prime}}^{\left(M, \nu_{M-1}\right)}\right\rangle,
\end{aligned}
$$

and the differential overlap

$$
\boldsymbol{\tau}_{j J, j^{\prime} J^{\prime}}^{\left(M, \nu_{M-1}\right)}=\delta_{j j^{\prime}} \tau_{J J^{\prime}}^{\left(M, \nu_{M-1}\right)}
$$

Finally, the equations of motion for the Gaussian parameters are again entirely analogous to those of G-MCTDH and 2L-GMCTDH, cf. Eq. (18),

$$
i \boldsymbol{C}^{\left(M, \nu_{M}\right)} \dot{\Lambda}^{\left(M, \nu_{M}\right)}=\boldsymbol{Y}^{\left(M, \nu_{M}\right)}
$$

with

$$
\begin{aligned}
C_{j \alpha, j^{\prime} \beta}^{\left(M, \nu_{M}\right)} & =\rho_{j j^{\prime}}^{\left(M, \nu_{M}\right)}\left\langle\partial_{\alpha} g_{j}^{\left(M, \nu_{M}\right)}\left|\left(1-\widehat{P}^{\left(M, \nu_{M}\right)}\right)\right| \partial_{\beta} g_{j^{\prime}}^{\left(M, \nu_{M}\right)}\right\rangle, \\
Y_{j \alpha}^{\left(M, \nu_{M}\right)} & =\sum_{j^{\prime}}\left\langle\partial_{\alpha} g_{j}^{\left(M, \nu_{M}\right)}\left|\left(1-\widehat{P}^{\left(M, \nu_{M}\right)}\right) \widehat{H}_{j j^{\prime}}^{\left(M, \nu_{M}\right)}\right| g_{j^{\prime}}^{\left(M, \nu_{M}\right)}\right\rangle
\end{aligned}
$$

and the shorthand notation $\partial_{\alpha} g_{j}^{\left(M, \nu_{M}\right)}=\left(\partial g_{j}^{\left(M, \nu_{M}\right)} / \partial \Lambda_{j, \alpha}^{\left(M, \nu_{M}\right)}\right)$.

To summarize, the above ML-GMCTDH scheme expands the first, orthogonal layer of the 2L-GMCTDH approach into a hierarchical multi-layer representation, while the last layer is again of GWP type. In practice, the above scheme is a modified ML-MCTDH approach, where the last layer is represented by non-orthogonal GWP particles rather than conventional SPFs. The above equations have been derived in Ref. [34] in a slightly more general form, for arbitrary constraint operators, $\widehat{h}_{c}^{\left(m, \nu_{m}\right)}$.

\section{CHARGE TRANSFER HAMILTONIAN AND SPECTRAL DENSITY}

In the present study, we use the ML-GMCTDH scheme to carry out non-adiabatic dynamics calculations for a two-state LVC Hamiltonian ${ }^{53}$ describing charge separation in a fullereneoligothiophene $\left(\mathrm{C}_{60}-\mathrm{OT}_{4}\right)$ complex, ${ }^{43}$ as illustrated in Fig. 1a). (Here, $\mathrm{OT}_{4}$ stands for an 
oligomer with four thiophene monomer units.) As mentioned in Sec. I, this system represents a minimal model for charge transfer in a P3HT:PCBM donor-acceptor blend. ${ }^{39-41,51,58-60}$ The P3HT:PCBM material in a so-called bulk heterojunction architecture $^{39}$ is a paradigm system in organic photovoltaics applications, exhibiting ultrafast exciton dissociation. ${ }^{40,51}$ Even though the present model is a minimal realization, it is able to capture the prominent role of vibronic couplings in the charge separation dynamics. The model in the present form is a building block for more general aggregate Hamiltonians describing the P3HT:PCBM material. $^{44-46}$

The model Hamiltonian as specified below is constructed from supermolecular LR-TDDFT calculations using a suitable diabatization procedure as reported in Ref. [42]. The Hamiltonian includes intramolecular modes of the $\mathrm{C}_{60}$ and $\mathrm{OT}_{4}$ fragments $\left(\left\{x_{i}\right\}\right)$ in terms of a spectral density $J(\omega)$, along with an explicit representation of the $\mathrm{C}_{60}-\mathrm{OT}_{4}$ intermolecular distance mode $(R)$. In particular, it turns out that the $R$ mode exerts a non-negligible influence on the electronic coupling between the two fragments, within the diabatic representation. The determination of vibronic couplings from electronic structure information using the relative displacement of excited-state equilibrium geometries (or alternatively, FranckCondon gradients), can be found in Refs. [43-45] and is briefly recapitulated in the following subsection.

TABLE I. Parameters, quoted in eV, for the $\widehat{H}_{S}$ and $\widehat{H}_{R}$ portions of the Hamiltonian, see Eqs. (42)-(43).

\begin{tabular}{ccccc}
\hline \hline$\Delta_{\mathrm{XT}-\mathrm{CT}}$ & $\gamma$ & $\gamma_{R}$ & $\kappa_{R}$ & $\omega_{R}$ \\
\hline 0.079 & 0.130 & -0.010 & 0.030 & 0.010 \\
\hline \hline
\end{tabular}




\section{A. Vibronic coupling Hamiltonian}

Within a generalized system-bath type set-up, the Hamiltonian for the oligothiophenefullerene complex under study takes the form

$$
\widehat{H}=\widehat{H}_{S}+\widehat{H}_{R}+\widehat{H}_{B}
$$

where $\widehat{H}_{S}$ refers to the electronic part, $\widehat{H}_{R}$ is the part of the Hamiltonian that depends on the inter-fragment $(R)$ coordinate, and $\widehat{H}_{B}$ corresponds to the "bath" Hamiltonian subsuming the intramolecular modes. Accounting for a two-dimensional electronic Hilbert space spanned by a photogenerated exciton state $|\mathrm{XT}\rangle$ localized on the oligothiophene moiety, and a charge transfer state $|\mathrm{CT}\rangle$, the $\widehat{H}_{S}$ part reads as follows,

$$
\widehat{H}_{S}=\Delta_{\mathrm{XT}-\mathrm{CT}}|\mathrm{CT}\rangle\langle\mathrm{CT}|+\gamma(|\mathrm{XT}\rangle\langle\mathrm{CT}|+| \mathrm{CT}\rangle\langle\mathrm{XT}|)
$$

where the XT-CT gap $\Delta_{\mathrm{XT}-\mathrm{CT}}$ and the electronic coupling $\gamma$ are of the order of $0.1 \mathrm{eV}$ (see Table I). Next, the part of the Hamiltonian relating to the inter-fragment mode, $\widehat{H}_{R}$, is given as

$$
\begin{aligned}
\widehat{H}_{R}=\frac{\omega_{R}}{2}\left(\widehat{R}^{2}+\widehat{P}^{2}\right)+\kappa_{R} \widehat{R}|\mathrm{CT}\rangle\langle\mathrm{CT}| \\
+\gamma_{R} \widehat{R}(|\mathrm{XT}\rangle\langle\mathrm{CT}|+| \mathrm{CT}\rangle\langle\mathrm{XT}|)
\end{aligned}
$$

where $\kappa_{R}$ and $\gamma_{R}$ are electronically diagonal and off-diagonal vibronic couplings. Mass and frequency weighted coordinates are used throughout.

Finally, the bath Hamiltonian takes the standard form, for $N_{\text {bath }}=(N-1)$ modes (where the total number $N$ includes the $\widehat{R}$ mode),

$$
\widehat{H}_{B}=\sum_{i=1}^{N_{\text {bath }}} \frac{\omega_{i}}{2}\left(\widehat{x}_{i}^{2}+\widehat{p}_{i}^{2}\right)+\sum_{i=1}^{N_{\text {bath }}} \kappa_{i} \widehat{x}_{i}|\mathrm{CT}\rangle\langle\mathrm{CT}|
$$

noting that the vibronic couplings $\left\{\kappa_{i}\right\}$ relate to displacements $\left\{\Delta x_{i}^{\text {eq }}=\kappa_{i} / \omega_{i}\right\}$ of the CT state equilibrium geometry relative to the equilibrium geometry of the XT state, which is 
(a)

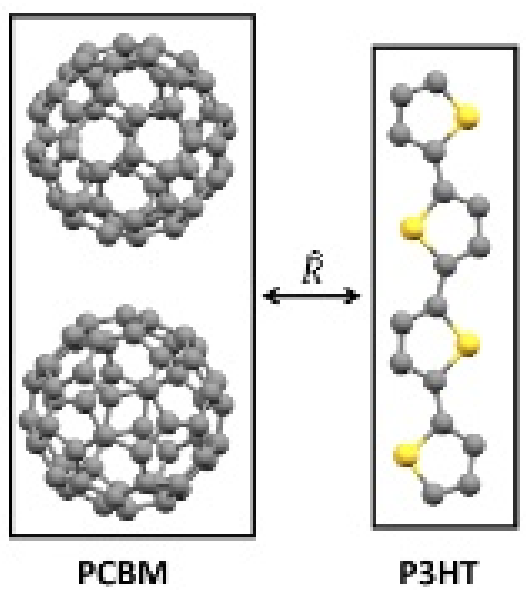

(b)

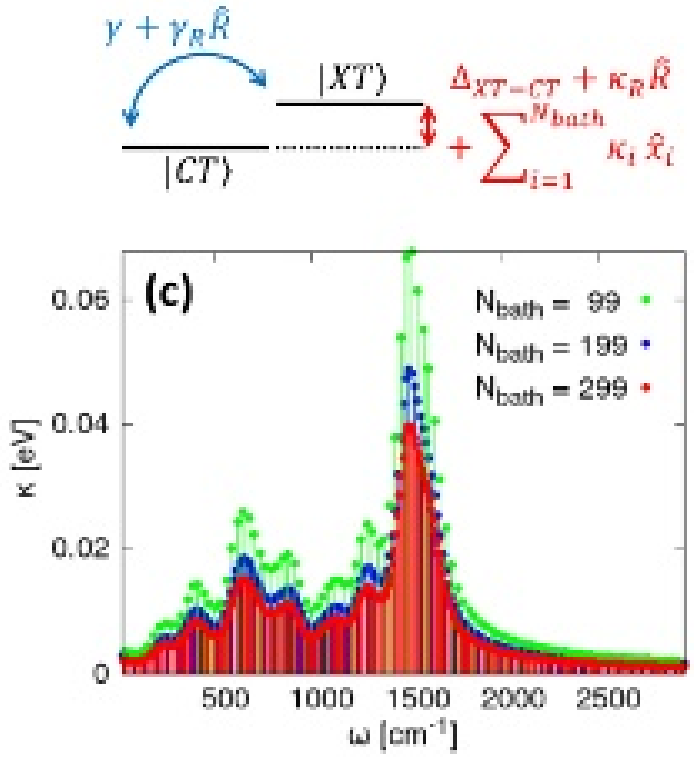

FIG. 1. (a) Schematic representation of a fullerene-oligothiophene $\left(\mathrm{C}_{60}-\mathrm{OT}_{4}\right)$ complex representative of a P3HT:PCBM interface. Here, the elementary building blocks are molecular units or else coarse-grained "super-particles" as indicated by the black frames. The inter-fragment coordinate $\widehat{R}$ entering the model is explicitly shown. (b) Sketch of the Hamiltonian model for the fullereneoligothiophene complex according to Eqs. (41)-(44). (c) Vibronic couplings $\kappa[\mathrm{eV}]$ for a discretized distribution of intramolecular modes of the oligothiophene-fullerene complex described in Ref. [ 43]. Vibronic couplings are shown for three discretized realizations, for $N_{\text {bath }}=99, N_{\text {bath }}=199$, and $N_{\text {bath }}=299$.

taken as reference. (In further detail, it is assumed in the present, simplified model that the Franck-Condon geometry coincides with the XT state equilibrium geometry. ${ }^{42,43}$ ) A sketch of the model Hamiltonian is shown in Fig. 1b).

\section{B. Spectral density}

A discretized spectral density was initially constructed for a $\mathrm{C}_{60}-\mathrm{OT}_{4}$ supermolecular complex. ${ }^{42}$ To this end, the normal modes (i.e., $N_{0}=246$ modes) of the charge-separated CT state were referred to, and the displacements $\Delta x_{i}^{\text {eq }}$ between the XT vs. CT state minima 
along the individual modes were computed. The vibronic couplings $\left\{\kappa_{i}\right\}$ and mode frequencies $\left\{\omega_{i}\right\}$ define a discretized spectral density which was subsequently convoluted with a Lorentzian broadening function,

$$
\begin{aligned}
J(\omega) & =\frac{\pi}{2} \sum_{i=1}^{N_{0}} \kappa_{i}^{2} \delta\left(\omega-\omega_{i}\right) \\
& \simeq \frac{\pi}{2} \sum_{i=1}^{N_{0}} \frac{\kappa_{i}^{2}}{\pi} \frac{\Delta}{\left(\omega-\omega_{i}\right)^{2}+\Delta^{2}}
\end{aligned}
$$

where the parameter $\Delta$ determines the Lorentzian width. In the calculations reported below, $\Delta=0.25 \Delta_{0}$ is used, where $\Delta_{0}$ corresponds to the root mean square (RMS) of the frequency spacings of the original data set. (See Ref. [43] for a detailed discussion of the effect of the $\Delta$ parameter.) The resulting continuous SD shows large amplitudes around $0.2 \mathrm{eV}(\sim$ $1600 \mathrm{~cm}^{-1}$ ), due to the characteristic $\mathrm{C}=\mathrm{C}$ and $\mathrm{C}=\mathrm{S}$ stretching modes of the $\mathrm{C}_{60}^{-}$and $\mathrm{OT}_{4}^{+}$ fragments. Also, the lower-frequency range below $0.1 \mathrm{eV}\left(\sim 800 \mathrm{~cm}^{-1}\right)$ features pronounced contributions, mainly due to $\mathrm{OT}_{4}^{+}$in-plane bending modes and the radial fullerene $\mathrm{H}_{g}$ modes. In view of carrying out quantum dynamical calculations for a variable number of bath modes, the continuous spectral density is re-discretized for an arbitrary number of $N_{\text {bath }}$ bath modes,

$$
J(\omega)=\frac{\pi}{2} \sum_{i=1}^{N_{\text {bath }}} \kappa_{i}^{2} \delta\left(\omega-\omega_{i}\right)
$$

where the vibronic couplings are obtained as follows from the reference spectral density $J(\omega)$ of Eq. (45), given an equidistant sampling interval $\Delta \omega,{ }^{61}$

$$
\kappa_{i}=\left(\frac{2}{\pi} J\left(\omega_{i}\right) \Delta \omega\right)^{1 / 2}
$$

The sampling interval determines the Poincaré recurrence period $\tau_{p}=2 \pi / \Delta \omega$. In the following, we will consider several realizations of the spectral density, corresponding to $N_{\text {bath }}=99$, $N_{\text {bath }}=199$, and $N_{\text {bath }}=299$, featuring Poincaré times equal to $1135 \mathrm{fs}, 2249 \mathrm{fs}$, and $3380 \mathrm{fs}$, respectively. The relevant sets of vibronic couplings are illustrated in Fig. 1c). The different numbers of bath modes allows us to study the effect of the system size on the performance of the ML-GMCTDH simulations reported below. 

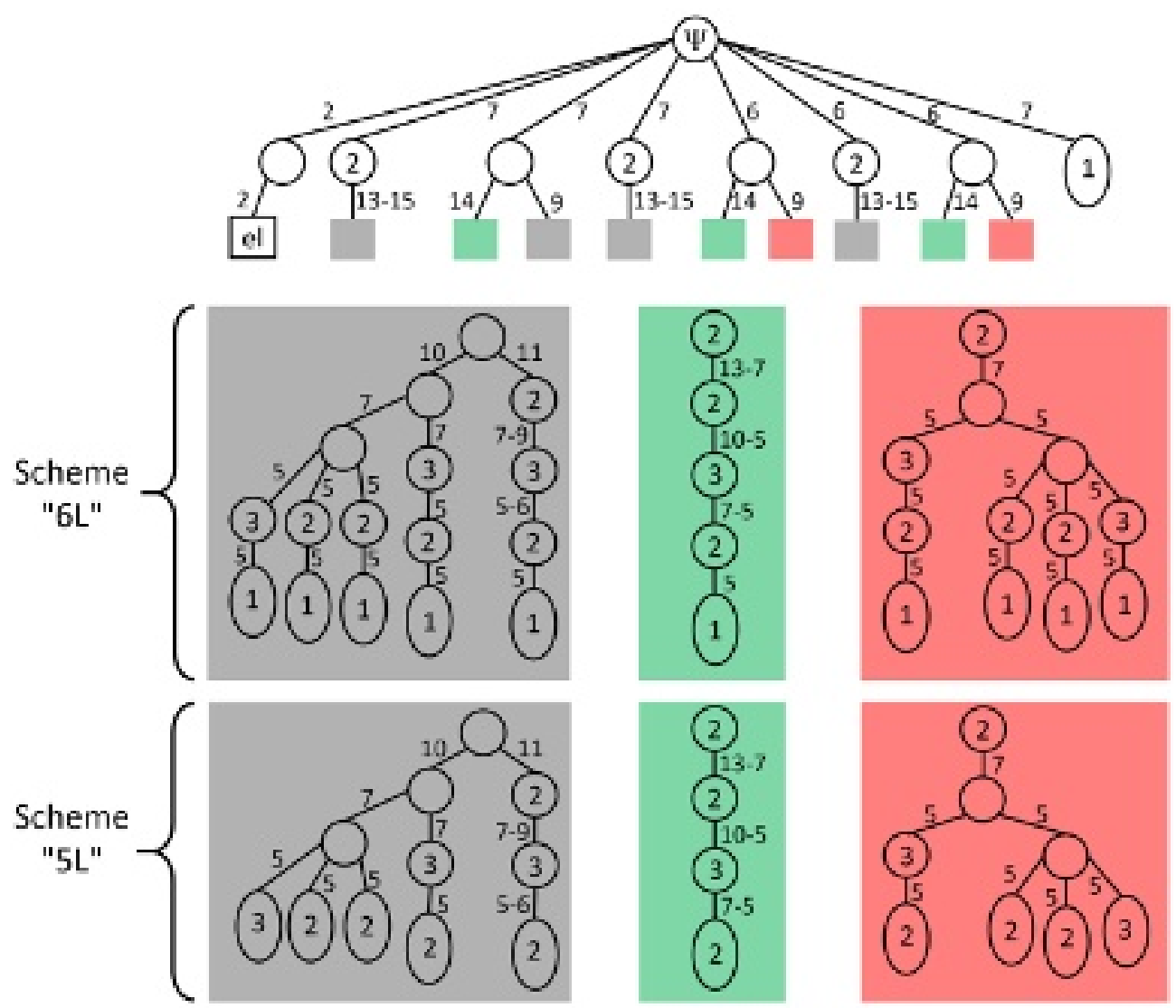

FIG. 2. Multi-layer tree structures featuring six layers $(6 \mathrm{~L})$ vs. five layers $(5 \mathrm{~L})$ employed for the 300-mode system. Circles represent nodes, whereas numbers next to lines connecting two nodes indicate the number of associated SPFs. Numbers placed inside a circle indicate that several identical replicas of the relevant sub-branch exist. If the equivalent sub-branches differ in the numbers of SPFs, we report the minimum and maximum numbers of SPFs used for the equivalent branches separated by a hyphen. As the tree is structured in a symmetrical fashion, equivalent sub-branches are highlighted in colored rectangles and are repeated as smaller colored boxes. The numbers indicated inside the ellipses give the number of combined modes. The electronic subbranch is denoted "el". The isolated branch on the r.h.s., with a single layer, relates to the intermolecular $\widehat{R}$ coordinate.

\section{ML-GMCTDH SIMULATION SET-UP AND PERFORMANCE}

In the following, we discuss results obtained with a new ML-GMCTDH implementation contained in the QUANTICS package. ${ }^{37}$ This implementation is more general than the 
TABLE II. Performance parameters for 100, 200, and 300-modes propagation schemes. The calculations were carried out on a 2.6 GHz Intel Xeon E5-2690v4 node (single-thread) with 256 GB memory.

\begin{tabular}{|c|c|c|c|c|c|}
\hline Number of & Multi-layer & & $\mathrm{CPU}$ & Number of & RAM \\
\hline modes & tree scheme & Method & time $[\mathrm{s}]$ & coefficients & {$[\mathrm{MB}]$} \\
\hline 100 & $6 \mathrm{~L}$ & ML-GMCTDH & 2223 & 46501 & 39 \\
\hline 100 & $6 \mathrm{~L}$ & ML-MCTDH & 1695 & 46501 & 16 \\
\hline 100 & $5 \mathrm{~L}$ & ML-GMCTDH & 1131 & 38776 & 37 \\
\hline 100 & $5 \mathrm{~L}$ & ML-MCTDH & 7999 & 38776 & 200 \\
\hline 200 & $6 \mathrm{~L}$ & ML-GMCTDH & 50299 & 604390 & 242 \\
\hline 200 & $6 \mathrm{~L}$ & ML-MCTDH & 43686 & 604390 & 142 \\
\hline 200 & $5 \mathrm{~L}$ & ML-GMCTDH & 26858 & 588440 & 240 \\
\hline 200 & $5 \mathrm{~L}$ & ML-MCTDH & 46243 & 588440 & 530 \\
\hline 300 & $6 \mathrm{~L}$ & ML-GMCTDH & 102969 & 1141294 & 327 \\
\hline 300 & $6 \mathrm{~L}$ & ML-MCTDH & 111897 & 1141294 & 269 \\
\hline 300 & $5 \mathrm{~L}$ & ML-GMCTDH & 57891 & 1117119 & 324 \\
\hline 300 & $5 \mathrm{~L}$ & ML-MCTDH & 86148 & 1117119 & 856 \\
\hline
\end{tabular}

previously available 2L-GMCTDH in-house code. ${ }^{35,36}$ The present implementation is entirely analogous to the ML-MCTDH code of the QUANTICS package (which is, in turn, analogous to the ML-MCTDH implementation of the Heidelberg MCTDH package ${ }^{62}$ ). Within this scheme, the last layer of the ML-MCTDH wavefunction branches has been replaced by GWPs as discussed above. Due to the LVC form of the potential and the normal-mode form of the kinetic energy, all matrix elements can be evaluated analytically and exactly as Gaussian moments. More generally, a local harmonic approximation (LHA) is employed for the potential matrix elements. ${ }^{16,24,35}$ 
In the integration scheme, regularization of the single-particle density matrices $\boldsymbol{\rho}$, which is mainly required due to initially unoccupied SPFs in the first $M-1$ layers, is carried out analogously to ML-MCTDH. Furthermore, the $\boldsymbol{S}$ and $\boldsymbol{C}$ matrices appearing in the last layer may incur numerical instabilities due to linear dependencies of the GWPs. For the $\boldsymbol{\rho}$ and $\boldsymbol{S}$ matrices, a standard regularization procedure is applied in line with previous applications of MCTDH, G-MCTDH, and vMCG, such that the eigenvalues below a pre-defined threshold value $\epsilon$ are adjusted as $\omega^{\text {reg }}=\omega+\epsilon \exp (-\omega / \epsilon)^{16,28,35}$ (where the regularization parameter typically takes the value $\epsilon=10^{-8}$ ). For the $\boldsymbol{C}$ matrix, a so-called dynamic coupling scheme ${ }^{16}$ is employed, by which the GWPs which give rise to linear dependencies are decoupled and evolve classically. Finally, the GWPs are renormalized at each step. In ML-GMCTDH, the linear dependency problem is generally alleviated due to the partitioning into subspaces; in the present applications, no significant numerical instabilities were noticeable.

Similarly to Ref. [36], initial conditions are constructed with a single occupied FG particle per subspace in the initial XT state. The unoccupied GWPs are defined by equidistant shifts in coordinate space for all modes; these coordinate shifts are defined such as to match a pre-defined GWP overlap (here, $S_{j l}=0.7$ ). In the present simulations, all GWPs share the same coherent-state width.

As explained above, we consider three rediscretizations of the spectral density shown in Fig. 1 , namely $N_{\text {bath }}=99(N=100), N_{\text {bath }}=199(N=200)$, and $N_{\text {bath }}=299(N=300)$. Reference calculations are carried out using the ML-MCTDH algorithm as implemented in the QUANTICS package, using the same multi-layer trees. In order to speed up the calculations, the Constant Mean Field (CMF) integrator is used; within this set-up, the Short Iterative Lanczos (SIL) algorithm is used for the top layer, and the fifth-order RungeKutta (RK5) algorithm is used for the lower layers. Results match with those obtained via the Variable Mean Field (VMF) integrator, up to deviations of time-dependent populations of the order of $10^{-2}$ (see Sec. S4 of the Supp. Mat.).

The multi-layer tree employed for the 300-mode calculations is shown in Figure 2; analogous multi-layer trees are illustrated in Sec. S1 of the Supp. Mat., for $N=100$ and $N=200$. The details of the mode combination scheme are described in Sec. S5 of the Supp. Mat. In 
general, a balanced multi-layer tree will lead to a favorable distribution of the numerical effort between the computation of the $m$ th-layer mean fields (along with the related effort of the solution to the coefficient equations), and the effort of GWP propagation. For the 2L-GMCTDH case, explicit expressions for the numerical effort were derived. ${ }^{34-36}$ While the constraints of the 2L-GMCTDH scheme led us to construct comparatively high-dimensional GWP subspaces in the 100-mode calculations for the same system that is studied here, ${ }^{36}$ the possibility to move to more than two layers now permits much lower-dimensional GWP spaces in the last layer, specifically comprising no more than one to three modes for $M=5$ or $M=6$ as detailed below.

As illustrated in Figure 2 for $N=300$, two variants of the multi-layer trees are employed: i.e., a six-layer $(6 \mathrm{~L})$ variant and a five-layer $(5 \mathrm{~L})$ variant. In the $6 \mathrm{~L}$ case, the GWP parameter propagation has been reduced to one-dimensional subspaces, whereas in the $5 \mathrm{~L}$ case, the GWPs are two-dimensional and sometimes three-dimensional. In both cases, the intermolecular $R$ mode, which is diagonally and off-diagonally correlated with the electronic subspace, is treated separately in terms of an isolated branch with a single layer of GWP type. Recalling that the propagation cost in the GWP subspaces is proportional to $(n d)^{3}$ where $n$ is the number of GWPs in the $\kappa$ th subspace and $d$ is the number of modes combined into a given FG mode (see Sec. II A 2), $d=2$ or $d=3$ in conjunction with $n=5$ remain favorable, such that the $5 \mathrm{~L}$ variant is overall preferable for large $N$ since the number of mean-field computations is reduced.

As can be seen from the numbers of SPFs and GWPs indicated in the multi-layer trees (typically between 5 and 15, see the numbers next to the edges of the graphs in Fig. 2), the dynamics are comparatively strongly correlated, even though less correlated than in the case of dynamics at conical intersections. ${ }^{32}$ The overall number of wavefunction coefficients are around $4.7 \times 10^{4}(6 \mathrm{~L})$ and $3.9 \times 10^{4}(5 \mathrm{~L})$ in the 100 -mode calculations, $6.0 \times 10^{5}(6 \mathrm{~L})$ and $5.9 \times 10^{5}(5 \mathrm{~L})$ in the 200 -mode calculations, and around $1.1 \times 10^{6}$ in the 300 -mode calculations (6L and 5L), see Table II. For all calculations, both ML-GMCTDH and ML-MCTDH, the maximum of the least populated natural orbital population remains below 1\% (see Sec. 7 of the Supp. Mat.). 

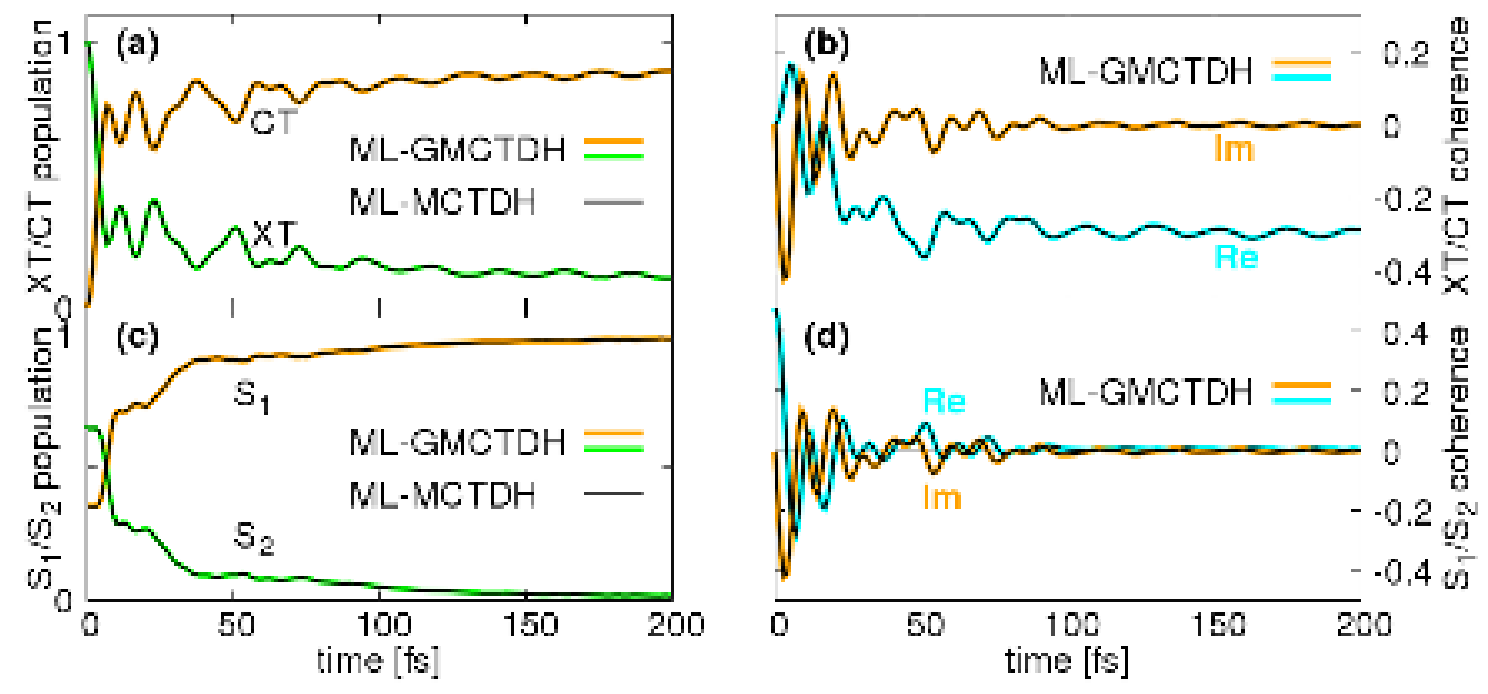

FIG. 3. Results obtained for ML-GMCTDH calculations for $N=300$ modes, where $N=N_{\text {bath }}+1$. The black lines correspond to ML-MCTDH reference calculations, while colored lines show MLGMCTDH results, here obtained with the $6 \mathrm{~L}$ scheme. a) Diabatic populations of the XT and CT states, b) real and imaginary parts of the electronic coherence, i.e., $\operatorname{Re} \rho_{\mathrm{XT}, \mathrm{CT}}$ and $\left.\operatorname{Im} \rho_{\mathrm{XT}, \mathrm{CT}}, \mathrm{c}\right)$ Adiabatic populations of the states $S_{1}$ and $S_{2}$, obtained by an approximate diabatic-to-adiabatic transformation. d) Likewise, real and imaginary parts of the adiabatic coherence, i.e., $\operatorname{Re} \rho_{S_{1}, S_{2}}$ and $\operatorname{Im} \rho_{S_{1}, S_{2}}$.

Table II further illustrates the numerical performance of the calculations as compared with reference ML-MCTDH calculations with the same ML tree structure (see Sec. S1 of the Supp. Mat. for further details). Briefly, in the reference calculations, the last-layer GWPs are replaced by conventional SPFs represented in a harmonic-oscillator discrete variable representation (HO-DVR), using 30 DVR points for each bath mode and 70 DVR points for the $R$ mode. In terms of CPU time, ML-GMCTDH calculations of 5L type always perform more favorably than their ML-MCTDH counterparts, and 6L type calculations become more favorable than ML-MCTDH as the system dimensionality increases to $N=300$. Among the ML-GMCTDH set-ups, 5L calculations are generally faster than $6 \mathrm{~L}$ calculations by a factor of two. This trend differs from the corresponding ML-MCTDH calculations, where the $6 \mathrm{~L}$ scheme is actually faster than the $5 \mathrm{~L}$ scheme by a factor of more than four for $N=100$, while the performance of the $5 \mathrm{~L}$ scheme becomes superior for $N=300$. Finally, the $5 \mathrm{~L}$ scheme is 
also more advantageous over ML-MCTDH as far as memory requirements go, at least by a factor of two (while the $6 \mathrm{~L}$ scheme seems to demand more memory than the corresponding ML-MCTDH calculations). Overall, mode combination is favorable in the last layer, but the multi-layer construction permits to avoid combining too many modes. By comparison, up to 8-dimensional FGs were constructed in our previous 2L-GMCTDH calculations for the same system, ${ }^{36}$ which were less efficient than the present ML-GMCTDH calculations.

\section{ML-GMCTDH SIMULATION RESULTS}

The present system has been chosen as an example of an ultrafast, coherent charge transfer process mediated by vibronic effects. From the perspective of Marcus theory, the system belongs to the inverted regime and features near-activationless charge separation. Yet, the ultrafast nature of the charge separation - observed as $\sim 50$ fs experimentally ${ }^{40,51}$ - cannot be rationalized by conventional rate theories. The present treatment explains the observed time scale and also highlights the role of several types of vibrational modes, notably the highfrequency $\mathrm{C}=\mathrm{C}$ (and $\mathrm{C}=\mathrm{S}$ ) stretch modes mentioned above, as well as the lower-frequency modes and the inter-fragment mode.

Since electronic and vibronic coherent effects play a prominent role in the dynamics described below, we computed time-evolving electronic coherences, i.e., off-diagonal elements of the electronic density matrix,

$$
\rho_{\mathrm{XT}, \mathrm{CT}}(t)=\operatorname{Tr}\{|\mathrm{CT}\rangle\langle\mathrm{XT}| \widehat{\rho}(t)\}
$$

where the trace $(\mathrm{Tr})$ is taken over the electronic and phonon degrees of freedom of the density operator, $\widehat{\rho}(t)=|\Psi(t)\rangle\langle\Psi(t)|$.

Figure 3 illustrates the charge separation dynamics in terms of diabatic and adiabatic state populations, along with the corresponding electronic coherences. Here, the adiabatic state populations and coherences were obtained by a simplified diabatic-to-adiabatic transformation along the paths defined by the full set of time-evolving coordinate expectation values (see Sec. S9 of the Supp. Mat.). The figure shows both ML-GMCTDH simulation results 


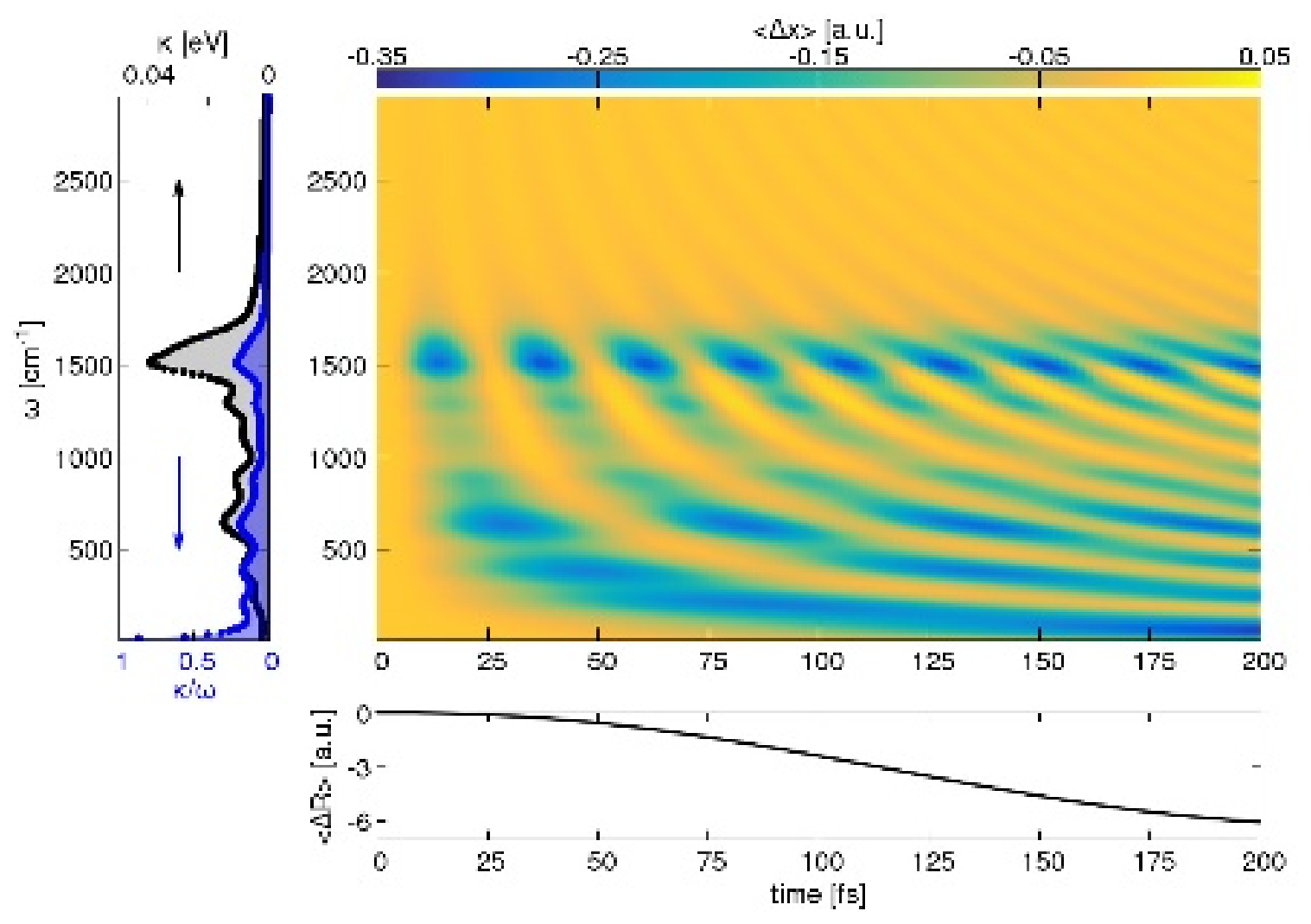

FIG. 4. Bath mode displacements (in atomic units) as a function of time and vibrational frequency. Results obtained at ML-GMCTDH level for 299 bath modes and adopting scheme 6L shown in Fig. 2. For clarity, in the left panel, we report the vibronic couplings $\kappa_{i}$ pertaining to the 299-mode bath spectral density shown in Fig. 1c, together with the equilibrium displacements $\Delta x_{i}^{\mathrm{eq}}=\left(\kappa_{i} / \omega_{i}\right)$. The time evolution of the intermolecular coordinate $R$ is shown in the bottom panel.

(using the 6L scheme) and ML-MCTDH reference calculations, and indicates no discernable difference between these simulations (see also the more detailed discussion below).

As detailed in Ref. [36 and 43], the dynamics can be considered to be driven by the electronic coherence, in a two-step process: First, the wave packet shows Rabi-type oscillations between the two diabatic states, given that the electronic coupling $\gamma$ and offset $\Delta_{\mathrm{XT}-\mathrm{CT}}$ are both of the order of $0.1 \mathrm{eV}$. In the process, a transient state-to-state population flux sets in, which is governed by the imaginary part $\operatorname{Im} \rho_{\mathrm{XT}, \mathrm{CT}}$ through the expression $\Gamma_{\mathrm{XT}, \mathrm{CT}}=(-2 \gamma / \hbar) \operatorname{Im} \rho_{\mathrm{XT}, \mathrm{CT}}{ }^{63,64}$ where $\gamma$ is the diabatic coupling (neglecting for simplicity the $R$-dependency of the coupling), see also Sec. S10 of the Supp. Mat. This flux goes to 
zero after about $50 \mathrm{fs}$, when the major part of the XT $\rightarrow$ CT transfer has happened (panels a) and b)). Second, the system relaxes to a quasi-stationary coherent superposition state as shown by the real part $\operatorname{Re} \rho_{\mathrm{XT}, \mathrm{CT}}$ which converges towards a constant value after the initial transient phase. This quasi-stationary state is essentially of CT character, with a small $(\sim 10 \%)$ excitonic component. ${ }^{43}$

From the complementary adiabatic picture (panels c) and d)), we equally infer that the initial non-adiabatic event exhibits coherent character during several tens of femtoseconds and results in a pure $S_{1}$ state, which is predominantly of charge transfer type but carries partial excitonic character. Note that the initial XT state corresponds to a superposition of the adiabatic $S_{1}$ and $S_{2}$ states, such that electronic and vibronic dynamics are intertwined during the first tens of femtoseconds. The computed ultrafast charge transfer dynamics, with an $S_{2}$ decay time $<50 \mathrm{fs}$, is in excellent agreement with the experimentally observed ultrafast exciton dissociation at P3HT:PCBM interfaces. ${ }^{40,51}$

To better understand why an extremely fast and apparently irreversible charge separation event takes place, it is essential to consider the role of vibronic coupling. In Fig. 4, the full set of vibrational displacements $\left\{\left\langle\Delta x_{i}\right\rangle(t)\right\}$ is shown as a function of time and vibrational frequency for $N_{\text {bath }}=299$. Similar results are obtained for the lower-dimensional rediscretizations of the bath spectral density, as shown in Sec. S3 of the Supp. Mat., including long-time simulations up to the Poincaré recurrence time. Due to the structure of the spectral density, collective oscillatory motions of groups of bath modes with similar frequency appear. These oscillations are found to persist beyond the time scale of the initial non-adiabatic transition ( $\sim 50 \mathrm{fs})$, as also observed experimentally. ${ }^{40,51}$ The amplitudes of the displacements reflect the shift in the equilibrium positions $\Delta x_{i}^{\mathrm{eq}}=\left(\kappa_{i} / \omega_{i}\right)$ (see left panel) of the individual modes in the CT state as compared with the XT reference state.

Given that the adiabatic energy gap $\left(\Delta_{S_{2}-S_{1}}=0.27 \mathrm{eV}\right)$ at the reference geometry, $\Delta x_{i}=0$, is significantly larger than the diabatic gap $\left(\Delta_{\text {XT-CT }}=0.079 \mathrm{eV}\right.$, see Table I), the highfrequency bath modes - with frequencies around $0.19 \mathrm{eV}$ - are expected to play an important role in the initial nonadiabatic transition. Further, a dense distribution of frequencies is essential to observe an irreversible decay. 

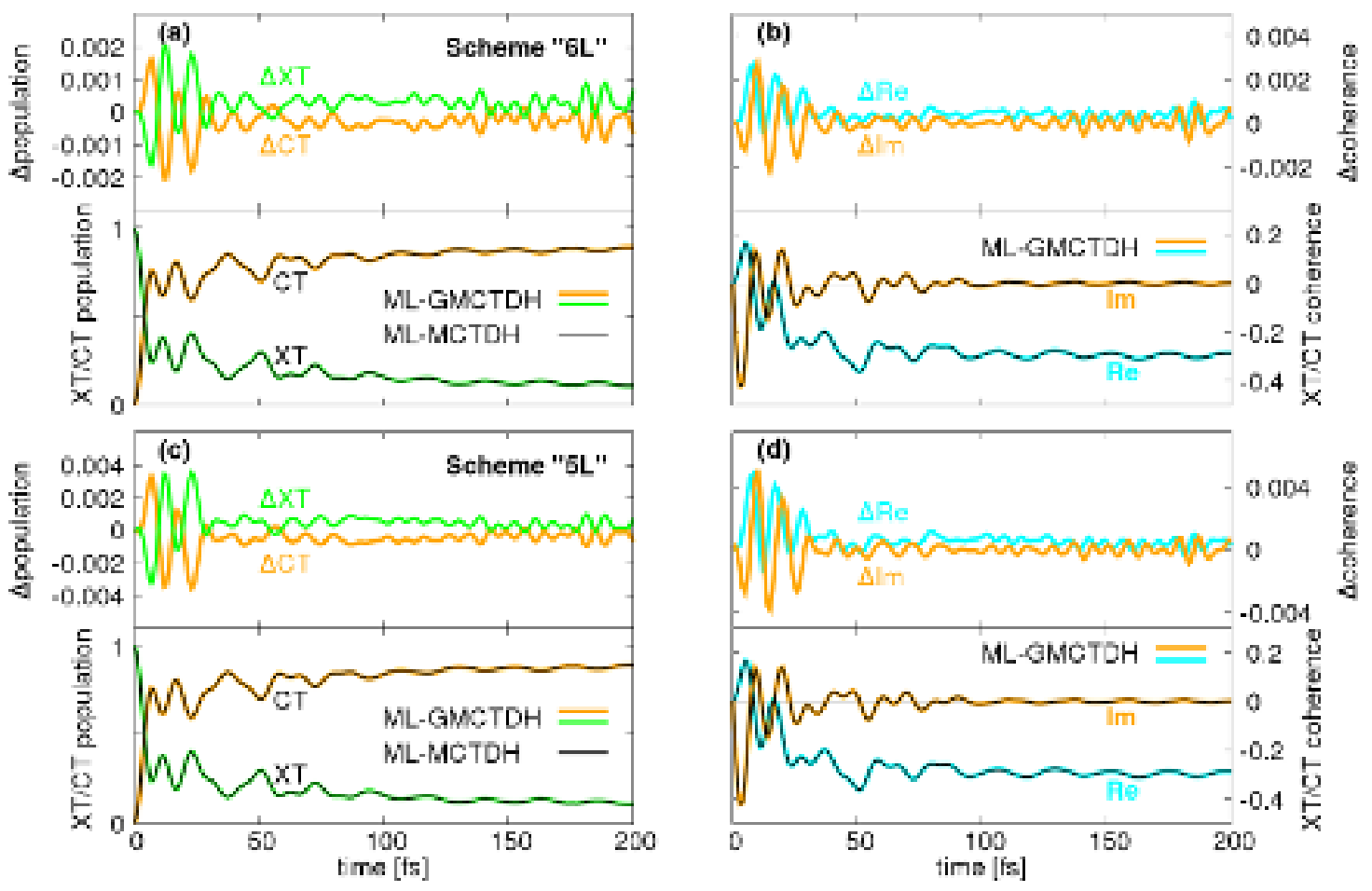

FIG. 5. Results obtained for ML-GMCTDH calculations for $N_{\text {bath }}=299$ modes, using scheme 6L (panels a) and b)) and scheme 5L (panels c) and d)). As in Fig. 3, ML-MCTDH reference calculations are shown as black lines. (Left panels) Time-evolution of the populations of the exciton (XT) state and of the charge transfer (CT) state. (Right panels) Real and imaginary parts of the electronic coherence $\rho_{\mathrm{XT}, \mathrm{CT}}$ as a function of time. The upper parts of each panel show the deviations from the ML-MCTDH reference calculations ( $\Delta$ population, $\Delta$ coherence) on a much larger scale.

Figure 4 also shows the time evolution of the inter-fragment coordinate $R$ (lower panel), which is found to move towards smaller values, indicating a contraction of the intermolecular distance due to the charge separation process. As discussed in further detail in Ref. [43] and Sec. S8 of the Supp. Mat., this leads to a significant change of both the diabatic energy gap and the diabatic coupling via the off-diagonal vibronic coupling $\gamma_{R}$ (see Eq. (43)), entailing a reduction of the coherent motions beyond the initial transients.

To conclude the present discussion, Fig. 5 compares 300-mode calculation results obtained with the $5 \mathrm{~L}$ vs. $6 \mathrm{~L}$ schemes, and the corresponding ML-MCTDH reference calculations. As already mentioned above, all calculations show excellent agreement, such that differences 
of time-evolving populations and coherences only become discernable on a scale of $10^{-3}$, as shown in the figure.

\section{CONCLUSIONS AND PERSPECTIVE}

The present study shows that variational moving Gaussian wavepackets can be conveniently and efficiently embedded into a hierarchical tensor representation of ML-MCTDH type. The resulting ML-GMCTDH approach has been implemented in the QUANTICS package, ${ }^{37}$ by adapting the last layer of the existing ML-MCTDH scheme to a non-orthogonal GWP representation. It should be noted that ML-GMCTDH retains all the properties of Gaussian bases that have ben used in the G-MCTDH and vMCG methods. Specifically, matrix elements can be calculated in terms of Gaussian moments, e.g., via local harmonic approximation, by Shepard interpolation, etc. In the present study the form of the potential allows the matrix elements to be calculated exactly analytically.

The QUANTICS implementation represents a generalization of the 2L-GMCTDH $(M=2)$ variant, which was previously employed to obtain converged propagation results for a 100mode realization of the same charge transfer system. ${ }^{36}$ In the present work, realizations involving up to 300 modes were treated, for which ML-GMCTDH proved at least as fast and efficient as its ML-MCTDH counterpart - and, in fact, even faster for the most favorable multi-layer construction, with a CPU time of 16 hours (single-thread) for a 300-mode calculation over $200 \mathrm{fs}$, as compared with 24 hours for the corresponding ML-MCTDH calculation. The ML-GMCTDH results are of high accuracy, and memory requirements tend to be favorable. In general, the performance of the method will depend on the number of GWPs - as compared with standard SPFs - that are required to accurately describe the dynamics of the last layer. As a rule of thumb, weakly coupled modes can be combined into multi-dimensional GWPs in the last layer, while strong correlations are naturally handled by the multi-layer structure.

The vibronic model Hamiltonian employed in the present study is a basic example of firstprinciples parametrized lattice Hamiltonians that can be expanded to much larger system 
sizes $^{44-46}$ in order to permit a full quantum treatment of photoinduced dynamics in molecular materials. Excited-state dynamics in these systems are characterized by electronic delocalization and strong electron-phonon (vibronic) coupling, both of which require accurate quantum dynamical approaches. Long-lived coherent vibronic features as observed in the present model complex indeed appear in spatially extended systems with dense electronic manifolds, too. ${ }^{40,51,65}$ As underscored by our previous studies, ${ }^{11,52}$ more approximate quantum-classical approaches may fail for this type of systems. For these reasons, on-the-fly dynamics for these systems, which is often restricted to Ehrenfest molecular dynamics ${ }^{66}$ or Surface-Hopping dynamics ${ }^{67}$ remain highly challenging.

Even though the present system is described by a basic linear vibronic coupling model with a large number of displaced harmonic-oscillator modes, several effects make the observed dynamics non-trivial. First, an ultrafast, coherent charge transfer event takes place which leads to a stationary coherent superposition state. These features alone are sufficient to lead to a complete failure of simple mean-field methods, necessitating a highly correlated treatment. Furthermore, the observed dynamics is induced by vibronic effects, specifically due the ubiquitous high-frequency CC and CS stretch modes, along with lower-frequency modes that provide a dense distribution of states and an inter-fragment mode that modulates the electronic coupling. These features make the propagation demanding and representative of dynamics in more complex systems. Finally, we did not include temperature effects in the present analysis - relying on earlier work on this system which showed that the effects are negligible for the ultrafast nonadiabatic dynamics ${ }^{42}$ - but temperature does play a key role, e.g., for diffusive processes ${ }^{68}$ which can be advantageously treated in the ML-GMCTDH framework.

Overall, the present approach bridges the gap between moving GWP basis sets, which often lack accuracy and flexibility, and the powerful tensor approaches which have led to MCTDH, ML-MCTDH, and related schemes. Using Gaussian basis sets in conjunction with novel potential energy surface fitting strategies, including neural network approaches ${ }^{69}$ opens a flexible approach to the accurate quantum dynamical treatment of large systems. The ML-GMCTDH approach can be combined with an on-the-fly implementation ${ }^{16}$ and new 
approaches to adaptive basis sets, ${ }^{70}$ and prepares the ground for future hybrid approaches that optimally combine trajectory-based descriptions with both accurate potentials and accurate quantum dynamics.

\section{DATA AVAILABILITY STATEMENT}

The data that supports the findings of this study are available within this article and the supplementary material.

\section{SUPPLEMENTARY MATERIAL}

See supplementary material for details concerning the following aspects: complete set of multi-layer tree structures (Sec. S1), comparison of simulations with different bath sizes (Sec. S2), additional vibrational displacement maps (Sec. S3), comparison with the Variable Mean Field (VMF) integrator (Sec. S4), details of the 5L mode combination scheme (Sec. S5), detailed results for $N=100$ and $N=200$ modes (Sec. S6), convergence aspects (Sec. S7), role of the inter-molecular mode (Sec. S8), approximate diabatic-adiabatic transformation (Sec. S9), nonadiabatic transition flux (Sec. S10).

\section{ACKNOWLEDGMENTS}

We thank Pierre Eisenbrandt, Dominik Brey and Sebastian Lenz for their assistance with preliminary calculations. F. D. M. gratefully acknowledges a research grant by the Humboldt Foundation. The German-Israeli Foundation for Scientific Research and Development is acknowledged for support of this project under grant number GIF I-1337-302.5/2016.

\section{REFERENCES}

${ }^{1}$ E. J. Heller, J. Chem. Phys. 62, 1544 (1975). 
${ }^{2}$ E. J. Heller, The Semiclassical Way to Dynamics and Spectroscopy, Princeton University Press, 2018.

${ }^{3}$ S. Lee and E. J. Heller, J. Chem. Phys. 76, 3035 (1982).

${ }^{4}$ E. J. Heller, J. Chem. Phys. 75, 2923 (1981).

${ }^{5}$ R. D. Coalson and M. Karplus, J. Chem. Phys. 93, 3919 (1990).

${ }^{6}$ S. Sawada, R. Heather, B. Jackson, and H. Metiu, J. Chem. Phys. 83, 3009 (1985).

${ }^{7}$ S. Sawada and H. Metiu, J. Chem. Phys. 84, 227 (1986).

${ }^{8}$ M. Ben-Nun and T. J. Martínez, Chem. Phys. Lett. 57, 298 (1998).

${ }^{9}$ D. V. Shalashilin and M. S. Child, Chem. Phys. 304, 103 (2004).

${ }^{10}$ D. V. Shalashilin, J. Chem. Phys. 130, 244101 (2009).

${ }^{11}$ T. Ma, M. Bonfanti, P. Eisenbrandt, R. Martinazzo, and I. Burghardt, J. Chem. Phys. 149, 244107 (2018).

${ }^{12}$ G. A. Worth and I. Burghardt, Chem. Phys. Lett. 368, 502 (2003).

${ }^{13}$ G. A. Worth et al., Faraday Discuss. 127, 307 (2004).

${ }^{14}$ M. Ben-Nun and T. J. Martínez, Adv. Chem. Phys. 121, 439 (2002).

${ }^{15}$ A. M. Virshup et al., J. Phys. Chem. B 113, 3280 (2009).

${ }^{16}$ G. Richings et al., Int. Rev. Phys. Chem. 34, 269 (2015).

${ }^{17}$ G. A. Worth, M. A. Robb, and I. Burghardt, Faraday Discuss. 127, 307 (2004).

${ }^{18}$ G. A. Worth, M. A. Robb, and B. Lasorne, Mol. Phys. 106, 2077 (2008).

${ }^{19}$ D. V. Makhov, C. Symonds, S. Fernandez-Alberti, and D. V. Shalashilin, Chem. Phys. 493, 200 (2017).

${ }^{20}$ M. Wehrle, S. Oberli, and J. Vaníček, J. Phys. Chem. A 119, 5685 (2015).

${ }^{21}$ D. J. Coughtrie and D. P. Tew, J. Chem. Phys. 143, 044102 (2015).

${ }^{22}$ I. Burghardt, M. Nest, and G. A. Worth, J. Chem. Phys. 119, 5364 (2003).

${ }^{23}$ S. Römer and I. Burghardt, Mol. Phys. 111, 3618 (2013).

${ }^{24}$ I. Burghardt, H.-D. Meyer, and L. S. Cederbaum, J. Chem. Phys. 111, 2927 (1999).

${ }^{25}$ I. Burghardt, K. Giri, and G. A. Worth, J. Chem. Phys. 129, 174104 (2008).

${ }^{26}$ H.-D. Meyer, U. Manthe, and L. Cederbaum, Chem. Phys. Lett. 165, 73 (1990).

${ }^{27}$ U. Manthe, H.-D. Meyer, and L. S. Cederbaum, J. Chem. Phys. 97, 3199 (1992).

${ }^{28}$ M. H. Beck, A. Jäckle, G. A. Worth, and H.-D. Meyer, Phys. Rep. 324, 1 (2000). 
${ }^{29}$ H. Wang and M. Thoss, J. Chem. Phys. 119, 1289 (2003).

${ }^{30}$ H. Wang, J. Phys. Chem. A 119, 7951 (2015).

${ }^{31}$ U. Manthe, J. Chem. Phys. 128, 164116 (2008).

${ }^{32}$ O. Vendrell and H.-D. Meyer, J. Chem. Phys. 134, 044135 (2011).

${ }^{33}$ X. Xie et al., J. Chem. Phys. 151, 224101 (2019).

${ }^{34}$ S. Römer, M. Ruckenbauer, and I. Burghardt, J. Chem. Phys. 138, 064106 (2013).

${ }^{35}$ P. Eisenbrandt, M. Ruckenbauer, S. Römer, and I. Burghardt, J. Chem. Phys. 149, 174101 (2018).

${ }^{36}$ P. Eisenbrandt, M. Ruckenbauer, and I. Burghardt, J. Chem. Phys. 149, 174102 (2018).

${ }^{37}$ G. A. Worth et al., The QUANTICS Package, Version 1.1 (2015), University of Birmingham, Birmingham, UK.

${ }^{38}$ G. A. Worth, Comput. Phys. Commun. 248, 107040 (2020).

${ }^{39}$ N. S. Sariciftci, L. Smilowitz, A. J. Heeger, and F. Wudl, Science 258, 1474 (1992).

${ }^{40}$ C. J. Brabec et al., Chem. Phys. Lett. 340, 232 (2001).

${ }^{41}$ R. D. Pensack and J. B. Asbury, J. Phys. Chem. Lett. 1, 2255 (2010).

${ }^{42}$ H. Tamura, I. Burghardt, and M. Tsukada, J. Phys. Chem. C 115, 10205 (2011).

${ }^{43}$ H. Tamura, R. Martinazzo, M. Ruckenbauer, and I. Burghardt, J. Chem. Phys. 137, 22A540 (2012).

${ }^{44}$ M. Polkehn, H. Tamura, and I. Burghardt, J. Phys. B 51, 014003 (2018).

${ }^{45}$ M. Polkehn, P. Eisenbrandt, H. Tamura, and I. Burghardt, Int. J. Quant. Chem. 118, e25502 (2018).

${ }^{46}$ W. Popp, D. Brey, R. Binder, and I. Burghardt, Quantum dynamics of exciton transport and dissociation in multi-chromophoric systems, Annu. Rev. Phys. Chem., vol. 72, in press (2021). doi: 10.1146/annurev-physchem-090419-040306, 2021.

${ }^{47}$ S. Mukamel, Principles of Nonlinear Optical Spectroscopy, Oxford University Press, New York/Oxford, 1995.

${ }^{48}$ K. H. Hughes, C. D. Christ, and I. Burghardt, J. Chem. Phys. 131, 124108 (2009).

${ }^{49}$ H. Tamura, J. Chem. Phys. 130, 214705 (2009).

${ }^{50}$ V. Tiwari, W. K. Peters, and D. M. Jonas, Proc. Nat. Acad. Sciences 110, 1203 (2013).

${ }^{51}$ A. De Sio et al., Eur. Phys. J. B 91, 236 (2018). 
${ }^{52}$ M. Bonfanti, G. A. Worth, and I. Burghardt, 2020, Multi-Configuration Time-Dependent Hartree Methods: From Quantum to Semiclassical and Quantum-Classical, in: Quantum Chemistry and Dynamics of Excited States: Methods and Applications, Eds. L. Gonzalez and R. Lindh, Wiley-Blackwell, ISBN: 978-1-119-41774-3 (2020).

${ }^{53}$ H. Köppel, W. Domcke, and L. S. Cederbaum, Adv. Chem. Phys. 57, 59 (1984).

${ }^{54}$ J. R. Klauder and B. S. Skagerstam, Coherent States, World Scientific, Singapore, 1985.

${ }^{55}$ D. V. Shalashilin and I. Burghardt, J. Chem. Phys. 129, 084104 (2008).

${ }^{56}$ C. Lubich, From Quantum to Classical Molecular Dynamics: Reduced Models and Numerical Analysis, European Mathematical Society Publishing House, Zuerich, Switzerland, 2008.

${ }^{57}$ I. R. Craig, H. Wang, and M. Thoss, J. Chem. Phys. 127, 144503 (2007).

${ }^{58}$ S. De et al., Chem. Phys. 350, 14 (2008).

${ }^{59}$ P. Parkinson, J. Lloyd-Hughes, M. B. Johnston, and L. M. Herz, Phys. Rev. B 78, 115321 (2008).

${ }^{60}$ R. D. Pensack, K. M. Banyas, L. W. Barbour, M. Hegadorn, and J. B. Asbury, Phys. Chem. Chem. Phys. 11, 2575 (2009).

${ }^{61}$ R. Martinazzo, K. H. Hughes, F. Martelli, and I. Burghardt, Chem. Phys. 377, 21 (2010).

${ }^{62}$ G. A. Worth, M. H. Beck, A. Jäckle, and H.-D. Meyer, The MCTDH Package, version 8.2, 2000; Meyer H.-D., version 8.3, 2002, and version 8.4, 2007. Version 8.4.14, 2017, used in this paper. http://mctdh.uni-hd.de/.

${ }^{63}$ Y. Tanimura and S. Mukamel, J. Chem. Phys. 101, 3049 (1994).

${ }^{64}$ I. Burghardt and L. S. Cederbaum, J. Chem. Phys. 115, 10312 (2001).

${ }^{65}$ A. S. Rury, S. A. Sorenson, and J. M. Dawlaty, J. Phys. Chem. Lett. 8, 181 (2017).

${ }^{66} \mathrm{Z}$. Xu et al., Nano Lett. 19, 8630 (2019).

${ }^{67}$ T. Nelson et al., Chem. Rev. 120, 2215 (2020).

${ }^{68}$ R. Binder and I. Burghardt, Faraday Discuss. 221, 406 (2020).

${ }^{69}$ W. Koch et al., J. Chem. Phys. 151, 064121 (2019).

${ }^{70}$ R. Martinazzo and I. Burghardt, Phys. Rev. Lett. 124, 150601 (2020). 


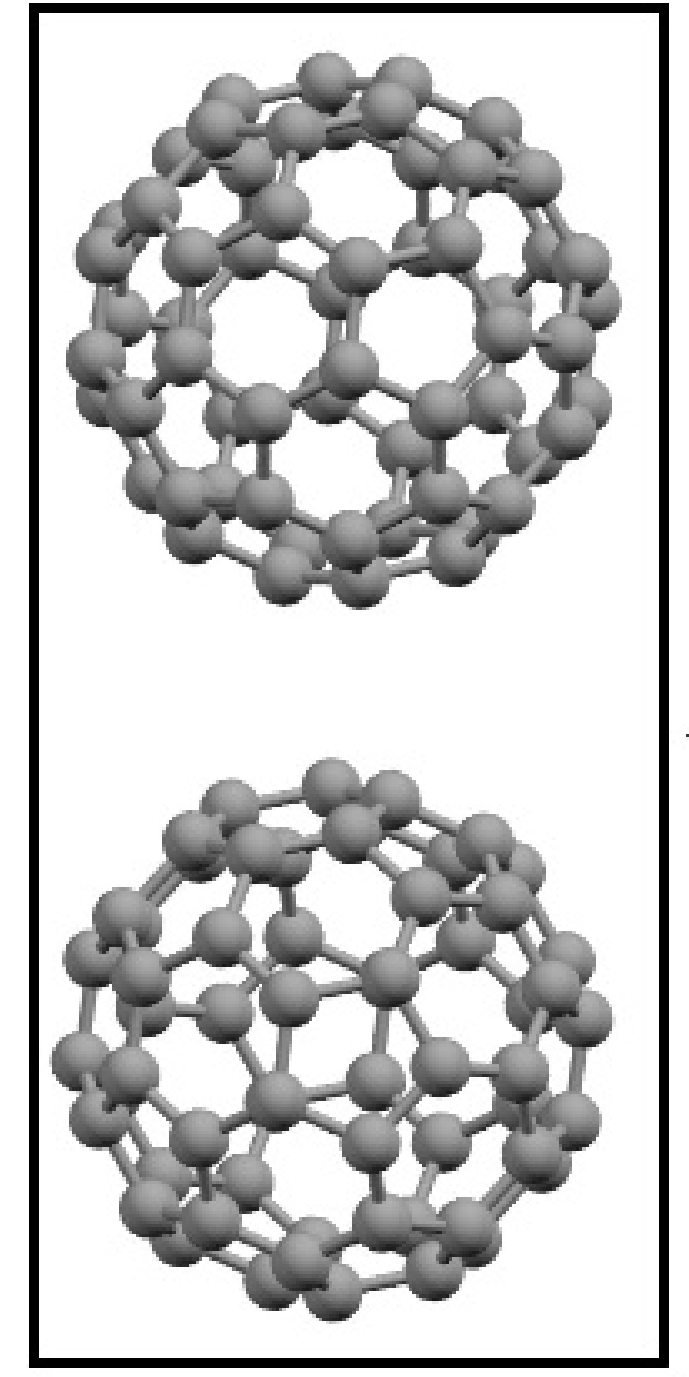

PCBM

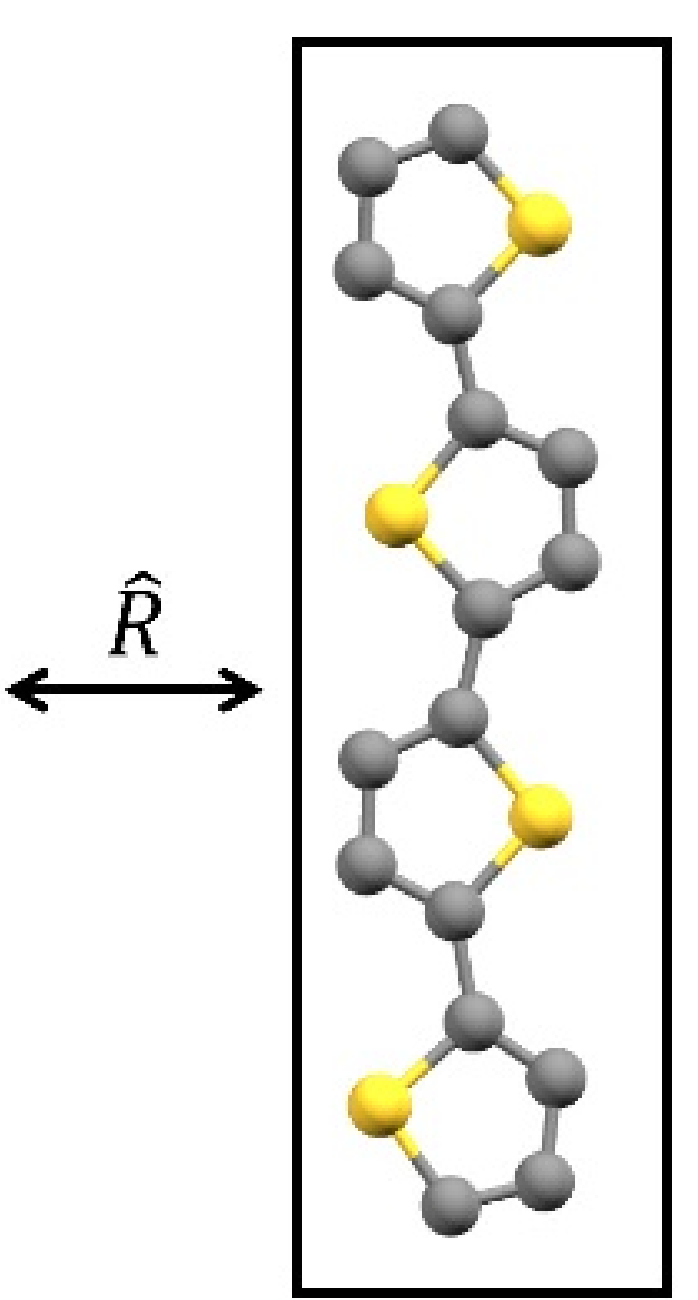

P3HT
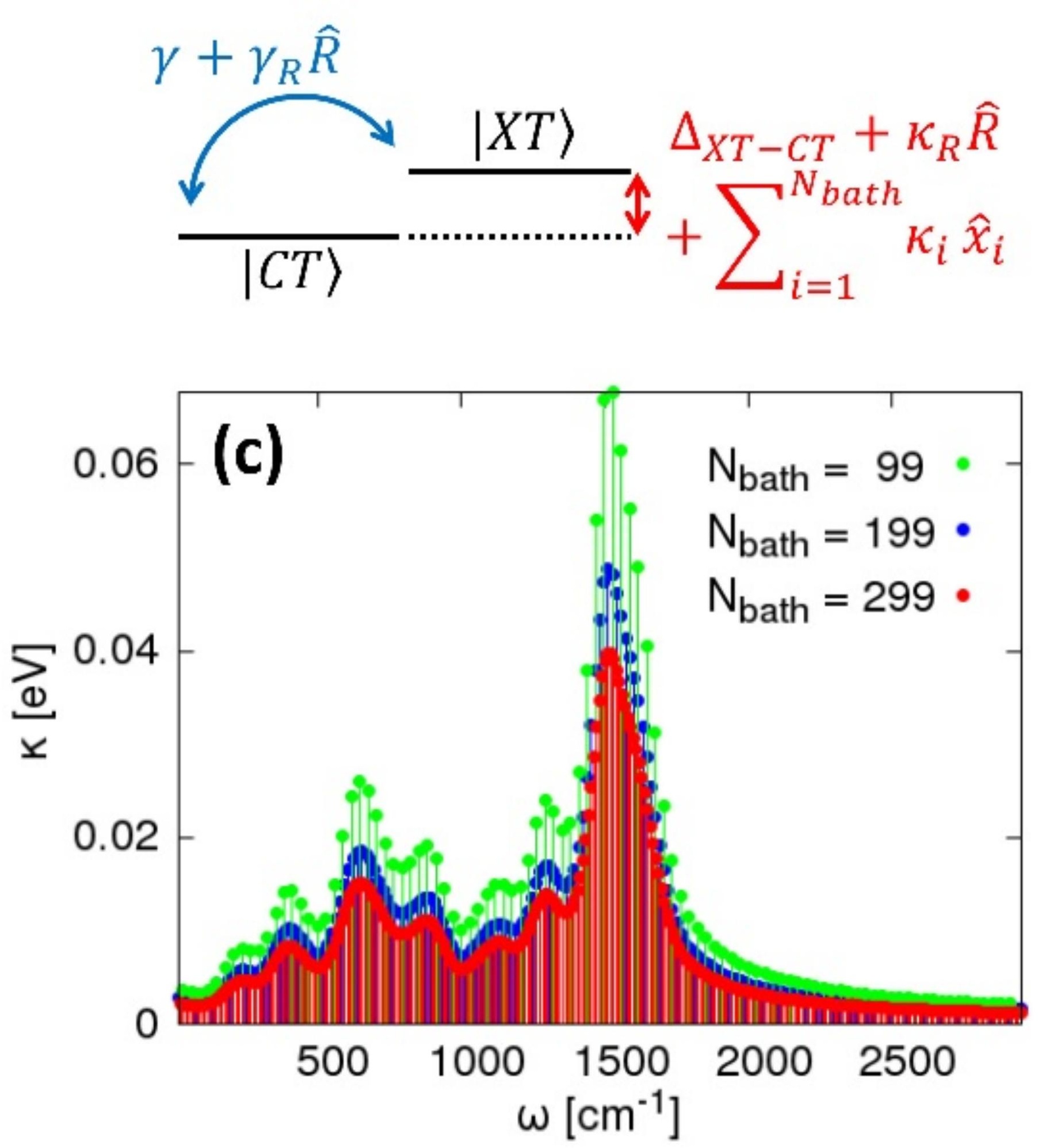

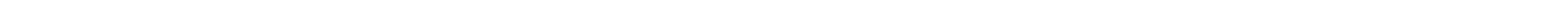


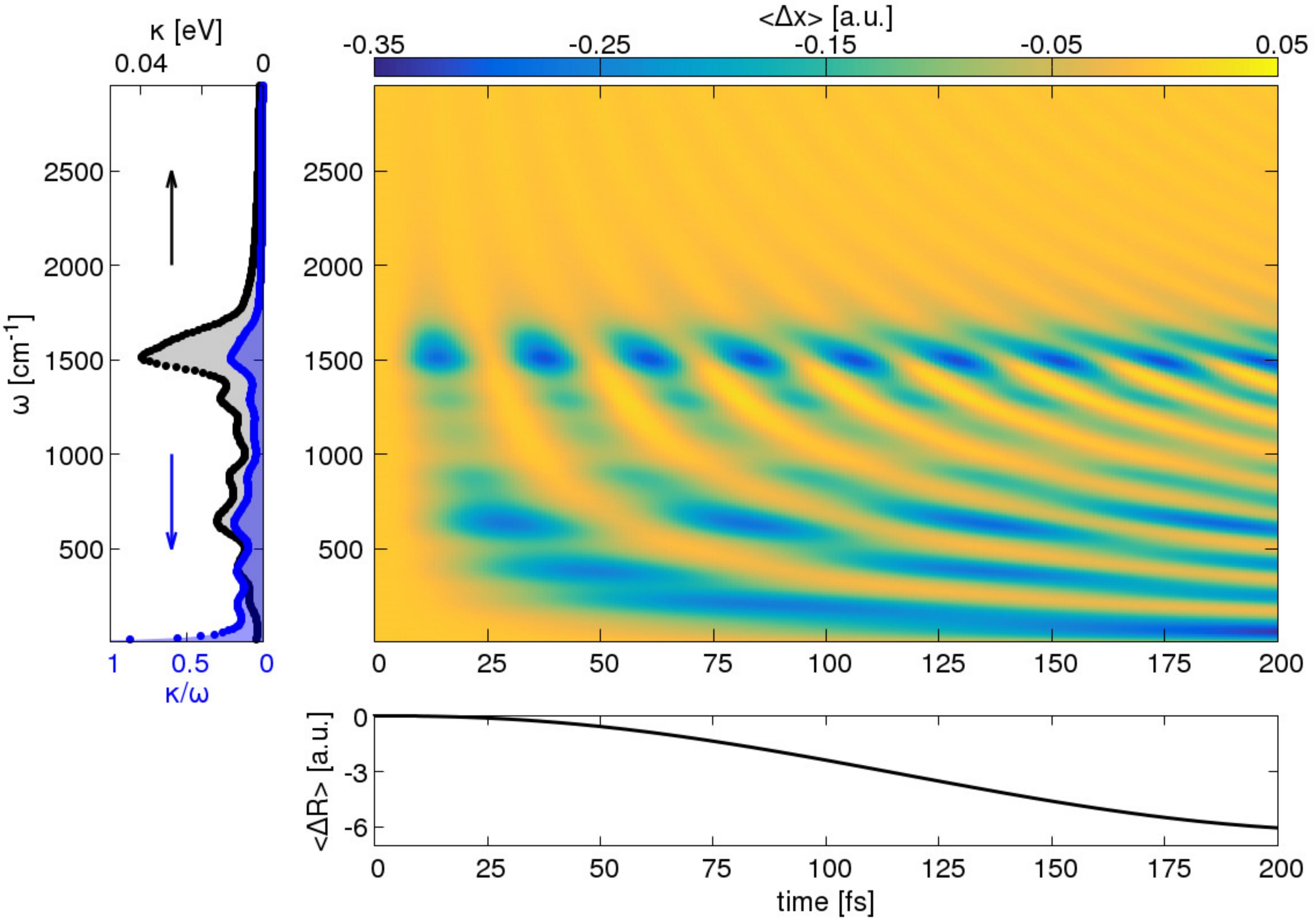




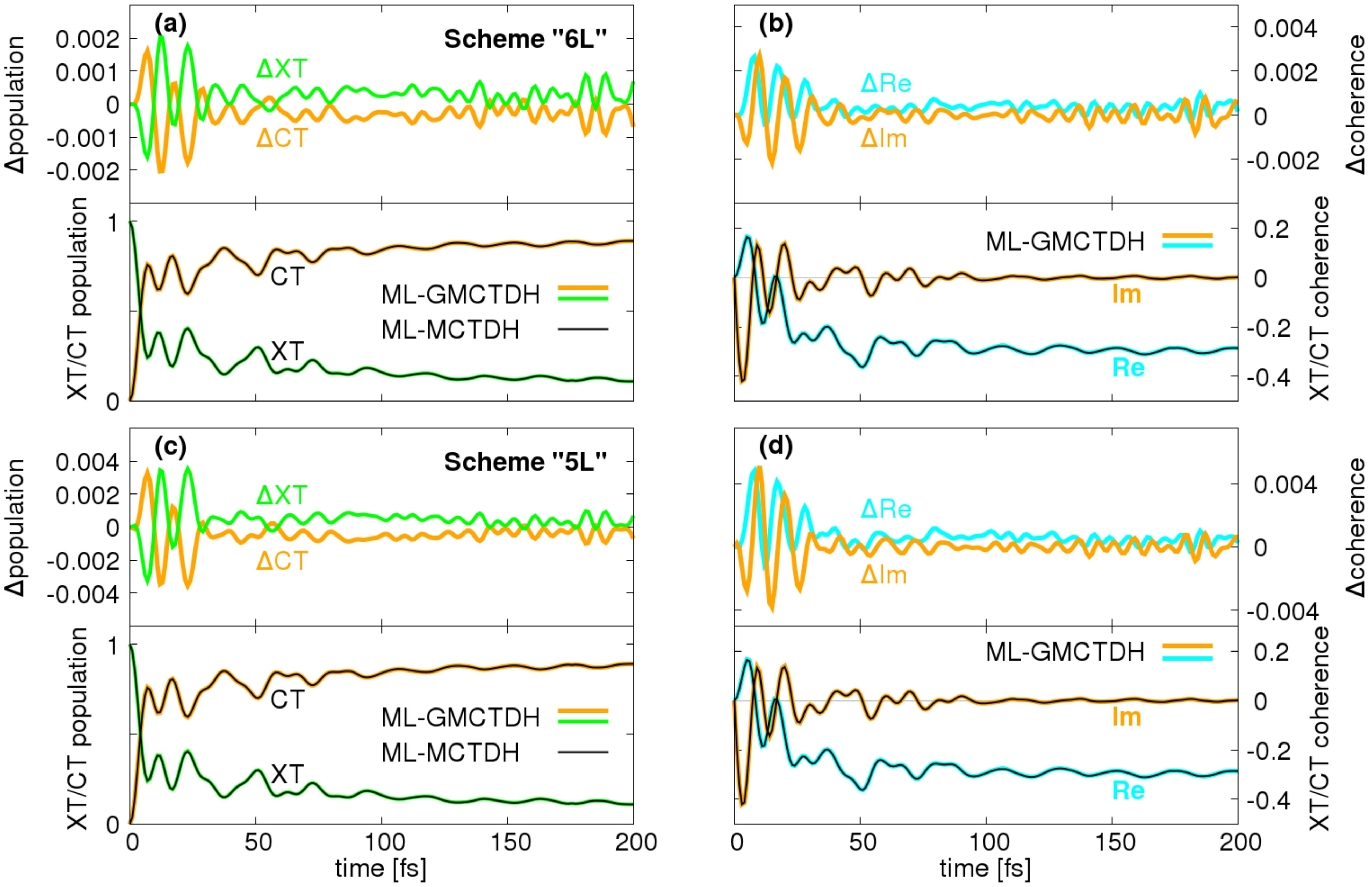

